THE Astrophysical Journal, 301:554-570, 1986 February 15

(C) 1986 The American Astronomical Society. All rights reserved. Printed in U.S.A.

\title{
RADIATIVE TRANSFER IN A SPHERE ILLUMINATED BY A PARALLEL BEAM: AN INTEGRAL EQUATION APPROACH ${ }^{1}$
}

\author{
RUN-LIE SHIA ${ }^{2}$ \\ Division of Physics, Mathematics, and Astronomy, California Institute of Technology \\ AND \\ YUK L. YUNG \\ Division of Geological and Planetary Sciences, California Institute of Technology \\ Received 1985 February 15 ; accepted 1985 June 4
}

\begin{abstract}
The problem of multiple scattering of nonpolarized light in a planetary body of arbitrary shape illuminated by a parallel beam is formulated using the integral equation approach. There exists a simple functional whose stationarity condition is equivalent to solving the equation of radiative transfer and whose value at the stationary point is proportional to the differential cross section. Our analysis reveals a direct relation between the microscopic symmetry of the phase function for each scattering event and the macroscopic symmetry of the differential cross section for the entire planetary body, and the inter-connection of these symmetry relations and the variational principle. The case of a homogeneous sphere containing isotropic scatterers is investigated in detail. It is shown that the solution can be expanded in a multipole series such that the general spherical problem is reduced to solving a set of decoupled integral equations in one dimension. Computations have been performed for a range of parameters of interest, and illustrative examples of applications to planetary problems are provided.
\end{abstract}

Subject headings: planets: atmospheres - radiative transfer

\section{INTRODUCTION}

The problem of radiative transfer in an object illuminated by a parallel beam is interesting both theoretically and practically. Extensive work has been performed for the plane-parallel atmosphere, and the results are summarized in the treatises by Chandrasekhar (1960), Sobolev (1975), and van de Hulst $(1980 a, b)$. But the problem with other geometries is more complicated (see review by Fouquart, Irvine, and Lenoble 1980). Recently, Flannery, Roberge, and Rybicki (1980) studied the radiative transfer of ultraviolet photons in a sphere and Chang and Kylafis (1983) investigated the scattering of X-rays in a spherical shell. In both cases the boundary conditions are spherically symmetric. The planetary problem with incident radiation from infinity has been given an invariant imbedding formulation (Bellman et al. 1969), but numerical results have been obtained only with Monte Carlo simulations (Modali, Brandt, and Kastner 1975; Anderson and Hord 1977; Adams and Kattawar 1978).

The approach we adopt is to use the integral equation and to exploit systematically its symmetry properties in order to simplify the mathematics. In this paper we report two initial successes of this approach. In the first part of the paper we provide the first complete formulation of the most general radiative transfer problem (except for polarization) in the form of a variational principle. We construct a functional and show that the equation of radiative transfer can be obtained by imposing the stationarity condition on this functional and that the differential cross section is given by the extremum of the functional. This result generalizes the work of Stokes and DeMarcus

\footnotetext{
${ }^{1}$ Contribution No. 4084 from the Division of Geological and Planetary Sciences, California Institute of Technology, Pasadena, California 91125.

${ }^{2}$ On leave from Institute of Theoretical Physics, Academia Sinica, Beijing, People's Republic of China.
}

(1971) and Chow, Friedson, and Yung (1984), and extends the work of Cheyney and Arking (1976). The symmetry of the phase function $P\left(\boldsymbol{\Omega}_{1}, \boldsymbol{\Omega}_{2}\right)=P\left(-\boldsymbol{\Omega}_{2},-\boldsymbol{\Omega}_{1}\right)$ (see detailed discussion in $\S$ III and Appendix C) plays a fundamental role in the variational principle.

In the second part of the paper the integral equation is applied to the simple case of a homogeneous sphere containing isotropic scatterers. We note that the symmetry of this problem is the same as that of the scattering of an electromagnetic wave by a dielectric sphere (Mie 1908; Debye 1909; van de Hulst 1957). The solution can, therefore, be expanded in a similar multipole series. This provides a natural generalization of the work of Sobolev (1972), whose solution corresponds to the first term of our series. Representative numerical results are presented, along with brief discussions of applications to planetary atmospheres.

\section{THE INTEGRAL EQUATION}

The integral equation for the specific intensity inside an object of volume $V$ and surface $S$ illuminated by a parallel beam is

$$
\begin{aligned}
I\left(\boldsymbol{r}_{1}, \mathbf{\Omega}_{1}\right)=F\left(\boldsymbol{r}_{1}, \mathbf{\Omega}_{1}\right)+\int_{0} \int_{4 \pi} d \boldsymbol{r}_{2} d \mathbf{\Omega}_{2} \delta\left(\mathbf{\Omega}_{1}-\hat{\boldsymbol{r}}_{12}\right) \\
\times \frac{e^{-\tau\left(\boldsymbol{r}_{1}, \boldsymbol{r}_{2}\right)}}{4 \pi r_{12}^{2}} \alpha\left(\boldsymbol{r}_{2}\right) P\left(\boldsymbol{r}_{2} ; \mathbf{\Omega}_{1}, \mathbf{\Omega}_{2}\right) I\left(\boldsymbol{r}_{2}, \mathbf{\Omega}_{2}\right),
\end{aligned}
$$

where $I(\boldsymbol{r}, \boldsymbol{\Omega})$ is the specific intensity at $\boldsymbol{r}$, in the direction $\boldsymbol{\Omega}$ in units of photons $\mathrm{cm}^{-2} \mathrm{~s}^{-1} \mathrm{sr}^{-1}, P\left(\boldsymbol{r} ; \boldsymbol{\Omega}_{2}, \boldsymbol{\Omega}_{1}\right)$ is the phase function which gives the fraction of photons scattered at $r$ from $\boldsymbol{\Omega}_{1}$ to $\boldsymbol{\Omega}_{2}$ and is dimensionless, and $\tau\left(\boldsymbol{r}_{1}, \boldsymbol{r}_{2}\right)=\int_{\boldsymbol{r}_{1}}^{\boldsymbol{r}_{2}} d s \alpha(\boldsymbol{r})$ is the optical depth between $\boldsymbol{r}_{1}$ and $\boldsymbol{r}_{2}$ and is dimensionless. Here $d s=|d \boldsymbol{r}|, \alpha(\boldsymbol{r})$ is the extinction coefficient in units of $\mathrm{cm}^{-1}$, $r_{12}=\left|\boldsymbol{r}_{1}-\boldsymbol{r}_{2}\right|$, and $\hat{\boldsymbol{r}}_{12}=\left(\boldsymbol{r}_{1}-\boldsymbol{r}_{2}\right) / r_{12}$. As illustrated in Figure 
$1 a$, equation (1) states that the specific intensity at $\boldsymbol{r}_{1}$ consists of two contributions. The first is derived from the primary solar radiation propagating from infinity to $V$ in direction $\boldsymbol{k}_{0}$,

$$
F\left(\boldsymbol{r}_{1}, \mathbf{\Omega}_{1}\right)=\pi F_{0} \delta\left(\mathbf{\Omega}_{1}-\boldsymbol{k}_{0}\right) \epsilon\left(\boldsymbol{r}_{1}, \boldsymbol{k}_{0}\right),
$$

where $\pi F_{0}$ is the solar flux in units of photons $\mathrm{cm}^{-2} \mathrm{~s}^{-1}$ (as defined in Chandrasekhar 1960), $\delta(\mathbf{\Omega})$ is a two-dimensional $\delta$-function given by

$$
\int d \mathbf{\Omega} \delta\left(\mathbf{\Omega}-\mathbf{\Omega}_{0}\right) F(\mathbf{\Omega})=F\left(\mathbf{\Omega}_{0}\right),
$$

and the attenuation factor is given by

$$
\epsilon\left(\boldsymbol{r}_{1}, \boldsymbol{k}_{0}\right)=e^{-\tau\left[\boldsymbol{r}_{1}, \boldsymbol{R}\left(\boldsymbol{r}_{1}, \boldsymbol{k}_{0}\right)\right]},
$$

with $\boldsymbol{R}\left(\boldsymbol{r}_{1}, \boldsymbol{k}_{0}\right)$ being the point on $S$ where the solar beam that intercepts $\boldsymbol{r}_{1}$ first enters $V$. Inspection of Figure $1 a$ reveals that

$$
\left[\boldsymbol{r}_{1}-\boldsymbol{R}\left(\boldsymbol{r}_{1}, \boldsymbol{k}_{0}\right)\right] \cdot \boldsymbol{k}_{0}=\left|\boldsymbol{r}_{1}-\boldsymbol{R}\left(\boldsymbol{r}_{1}, \boldsymbol{k}_{0}\right)\right| .
$$

The second term in equation (1) arises from photons which are scattered from all other points $\boldsymbol{r}_{2}$ into $\boldsymbol{r}_{1}$. The structure and meaning of equation (1) are either obvious or can be easily understood by referring to standard texts on transport theory (Davison 1957; Case and Zweifel 1967). A complete solution of equation (1) provides the most detailed information of the internal and external radiation field. For observations made at asymptotic distance from $V$, it is convenient to define a differential cross section for scattering of radiation from initial direc- tion $\boldsymbol{k}_{0}$ into final direction $\boldsymbol{k}$ :

$$
\frac{d \sigma}{d \Omega}\left(\boldsymbol{k}, \boldsymbol{k}_{0}\right)=\frac{1}{4 \pi^{2} F_{0}} \int_{0} \int_{4 \pi} d \boldsymbol{r} d \boldsymbol{\Omega} \alpha(\boldsymbol{r}) \epsilon(\boldsymbol{r},-\boldsymbol{k}) P(\boldsymbol{r} ; \boldsymbol{k}, \boldsymbol{\Omega}) I(\boldsymbol{r}, \boldsymbol{\Omega}) .
$$

Note that the attenuation factor is $\epsilon(\boldsymbol{r},-\boldsymbol{k})$ for a beam that leaves $V$ in the direction $\boldsymbol{k}$ (see Fig. $1 a$ ). The differential cross section is a fundamental physical quantity, and its relation to the more familiar quantities such as reflectivity and phase variation is discussed in Appendix B.

For most problems of practical interest, the phase function admits of a simple expansion:

$$
\begin{aligned}
P\left(\boldsymbol{r} ; \mathbf{\Omega}_{1}, \mathbf{\Omega}_{2}\right) & =\sum_{l=0}^{\infty} \omega_{l}(\boldsymbol{r}) P_{l}\left(\mathbf{\Omega}_{1} \cdot \mathbf{\Omega}_{2}\right) \\
& =\sum_{l=0}^{\infty} \omega_{l}(\boldsymbol{r}) \frac{4 \pi}{2 l+1} \sum_{m=-l}^{l} Y_{l m}\left(\mathbf{\Omega}_{1}\right) Y_{l m}^{*}\left(\mathbf{\Omega}_{2}\right) \\
& =4 \pi \sum_{l m} \frac{\omega_{l}(\boldsymbol{r})}{2 l+1} Y_{l m}\left(\mathbf{\Omega}_{1}\right) Y_{l m}^{*}\left(\mathbf{\Omega}_{2}\right)
\end{aligned}
$$

where $P_{l}$ and $Y_{l m}$ are Legendre polynomials and spherical harmonics, respectively, and $\omega_{0}(\boldsymbol{r})$ is single-scattering albedo. Unless otherwise stated, the summation over the indices $l$ and $m$ are over the range 0 to $\infty$ for $l$, and $-l$ to $l$ for $m$. Without loss of generality, the specific intensity and the solar term can

a)

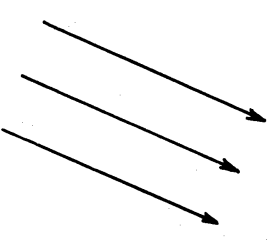

incident

beam k。

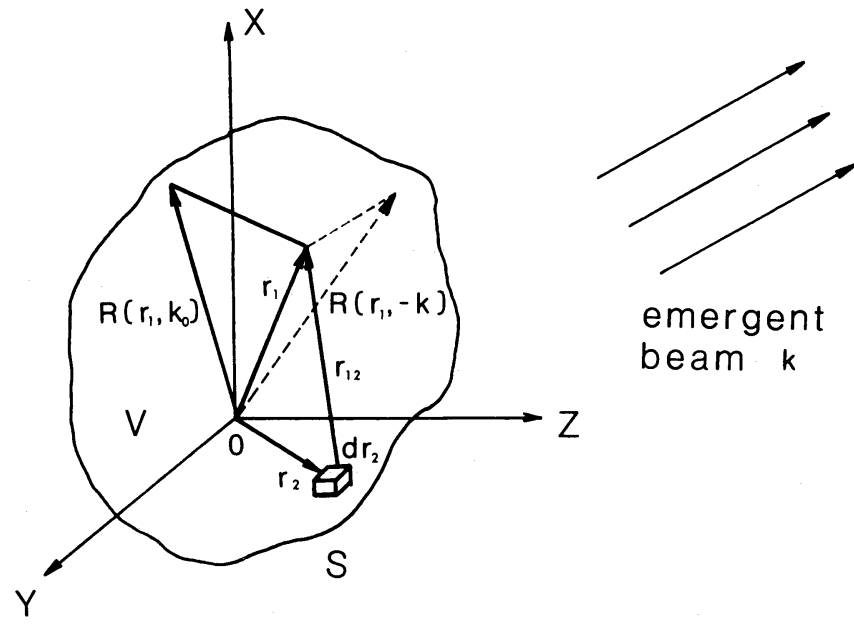

b)

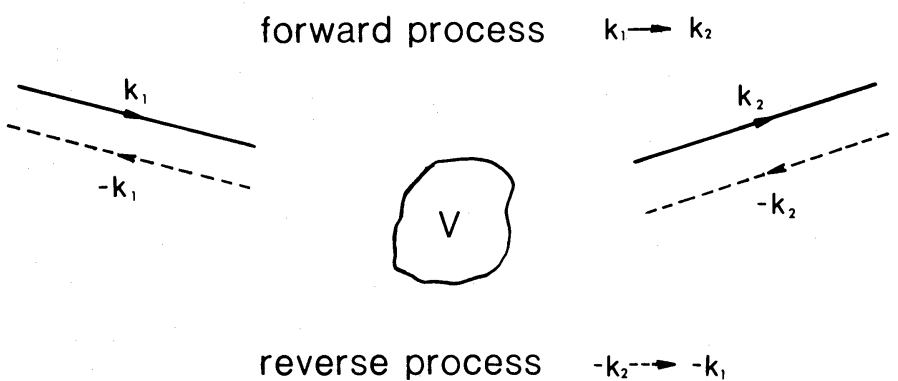

Fig. 1.- (a) Schematic diagram for radiative transfer in an object of volume $v$ and surface $S$. Incident and emergent beams are in the directions $\boldsymbol{k}_{0}$ and $\boldsymbol{k}$, respectively. Points $r_{1}$ and $r_{2}$ are interior points, and $d r_{2}$ is an infinitesmal volume at $r_{2}$, points $R\left(r_{1}, \boldsymbol{k}_{0}\right)$ and $R\left(\boldsymbol{r}_{1},-\boldsymbol{k}\right)$ are on the surface defined such that $R\left(\boldsymbol{r}_{1}, \boldsymbol{k}_{0}\right)-\boldsymbol{r}_{1}$ is antiparallel to $\boldsymbol{k}_{0}$ and $R\left(\boldsymbol{r}_{1},-\boldsymbol{k}\right)-\boldsymbol{r}_{1}$ is parallel to $\boldsymbol{k}$. (b) Schematic diagram for scattering of sunlight by an object of volume $v$. In the forward process the incident and observation directions are $\boldsymbol{k}_{1}$ and $\boldsymbol{k}_{2}$, respectively. In the reverse process the corresponding directions are $-\boldsymbol{k}_{2}$ and $-\boldsymbol{k}_{1}$, respectively. 
be expanded as follows:

$$
\begin{aligned}
& I(\boldsymbol{r}, \boldsymbol{\Omega})=\sum_{l m}\left[\frac{\alpha(\boldsymbol{r}) \omega_{l}(\boldsymbol{r})}{2 l+1}\right]^{-1 / 2} J_{l m}(\boldsymbol{r}) Y_{l m}(\mathbf{\Omega}), \\
& F(\boldsymbol{r}, \mathbf{\Omega})=\sum_{l m}\left[\frac{\alpha(\boldsymbol{r}) \omega_{l}(\boldsymbol{r})}{2 l+1}\right]^{-1 / 2} F_{l m}(\boldsymbol{r}) Y_{l m}(\mathbf{\Omega}) .
\end{aligned}
$$

Substituting equations (6), (7), and (8) into equation (1), multiplying equation (1) by $Y_{l_{1} m_{1}}^{*}\left(\boldsymbol{\Omega}_{1}\right)$, and integrating over $d \boldsymbol{\Omega}_{1}$, we obtain a set of coupled integral equations:

$J_{l_{1} m_{1}}\left(\boldsymbol{r}_{1}\right)=F_{l_{1} m_{1}}\left(\boldsymbol{r}_{1}\right)+\int_{0} d \boldsymbol{r}_{2} \sum_{l_{2} m_{2}} K_{l_{1} m_{1} l_{2} m_{2}}\left(\boldsymbol{r}_{1}, \boldsymbol{r}_{2}\right) J_{l_{2} m_{2}}\left(\boldsymbol{r}_{2}\right)$,

where

$$
\begin{array}{r}
F_{l_{1} m_{1}}\left(\boldsymbol{r}_{1}\right)=\left[\frac{\alpha\left(\boldsymbol{r}_{1}\right) \omega_{l_{1}}\left(\boldsymbol{r}_{1}\right)}{2 l_{1}+1}\right]^{1 / 2} \pi F_{0} \epsilon\left(\boldsymbol{r}_{1}, \boldsymbol{k}_{0}\right) Y_{l_{1} m_{1}}^{*}\left(\boldsymbol{k}_{0}\right), \\
K_{l_{1} m_{1} l_{2} m_{2}}\left(\boldsymbol{r}_{1}, \boldsymbol{r}_{2}\right)=\left[\frac{\omega_{l_{1}}\left(\boldsymbol{r}_{1}\right) \omega_{l_{2}}\left(\boldsymbol{r}_{2}\right) \alpha\left(\boldsymbol{r}_{1}\right) \alpha\left(\boldsymbol{r}_{2}\right)}{\left(2 l_{1}+1\right)\left(2 l_{2}+1\right)}\right]^{1 / 2} \frac{e^{-\tau\left(\boldsymbol{r}_{1}, \boldsymbol{r}_{2}\right)}}{r_{12}^{2}} \\
\times Y_{l_{1} m_{1}}^{*}\left(\hat{\boldsymbol{r}}_{12}\right) Y_{l_{2} m_{2}}\left(\hat{\boldsymbol{r}}_{12}\right) .
\end{array}
$$

Following Cheyney and Arking (1976), we rewrite equation (9) in a compact operator notation:

$$
(1-K) J=F,
$$

where

$$
\begin{aligned}
& J=\left[\begin{array}{llll}
J_{00}(\boldsymbol{r}), & J_{11}(\boldsymbol{r}), & J_{10}(\boldsymbol{r}), & J_{1-1}(\boldsymbol{r}), \cdots J_{l m}(\boldsymbol{r}), \cdots
\end{array}\right], \\
& F=\left[\begin{array}{llll}
F_{00}(\boldsymbol{r}), & F_{11}(\boldsymbol{r}), & F_{10}(\boldsymbol{r}), & F_{1-1}(\boldsymbol{r}), \cdots F_{l m}(\boldsymbol{r}), \cdots
\end{array}\right],
\end{aligned}
$$

and $K$ is the corresponding matrix. The operation of $K$ on $J$ involves both matrix multiplication and integration over spatial variable. Note that equation (12) is equivalent to equation (1). No approximation has been made.

\section{VARIATIONAL METHOD AND PRINCIPLE OF RECIPROCITY}

To employ the variational method for solving the integral equation of radiative transfer, the kernel of the equation must be self-adjoint. By definition the adjoint operator $K^{+}$is

$$
\begin{aligned}
K_{l_{1} m_{1} l_{2} m_{2}}^{+}\left(r_{1}, r_{2}\right)=K_{l_{2} m_{2} l_{1} m_{1}}^{*} & \left(r_{2}, r_{1}\right) \\
= & (-1)^{l_{1}+l_{2}} K_{l_{1} m_{1} l_{2} m_{2}}\left(\boldsymbol{r}_{1}, \boldsymbol{r}_{2}\right),
\end{aligned}
$$

where upper asterisk denotes complex conjugation. In general, $K$ is not self-adjoint; i.e., $K^{+} \neq K$. However, for phase functions with symmetry properties given by equation (6) (we shall later show how to relax this requirement), it is possible to define an extended equation which includes equation (12), and whose operator is self-adjoint. In the following discussion the mathematics is adapted from Cheyney and Arking (1976), but the physical interpretation and insight are new.

Suppose we adopt a new coordinate system to describe the direction of the radiation. In the new system the direction $\boldsymbol{\Omega}$ in the original system becomes $-\boldsymbol{\Omega}$. The positive vector $\boldsymbol{r}$ is unchanged. We will use the tilde sign to denote quantities in the new system, for example:

$$
\begin{gathered}
\tilde{I}(\boldsymbol{r}, \boldsymbol{\Omega})=I(\boldsymbol{r},-\mathbf{\Omega}), \\
\tilde{F}(\boldsymbol{r}, \boldsymbol{\Omega})=F(\boldsymbol{r},-\boldsymbol{\Omega}) .
\end{gathered}
$$

Expanding $\tilde{I}$ and $\tilde{F}$ we have

$$
\begin{aligned}
& \tilde{I}(\boldsymbol{r}, \boldsymbol{\Omega})=\sum_{l m}\left[\frac{\alpha(\boldsymbol{r}) \omega_{l}(\boldsymbol{r})}{2 l+1}\right]^{-1 / 2} \tilde{J}_{l m}(\boldsymbol{r}) Y_{l m}(\boldsymbol{\Omega}), \\
& \tilde{F}(\boldsymbol{r}, \boldsymbol{\Omega})=\sum_{l m}\left[\frac{\alpha(\boldsymbol{r}) \omega_{l}(\boldsymbol{r})}{2 l+1}\right]^{-1 / 2} \tilde{F}_{l m}(\boldsymbol{r}) Y_{l m}(\boldsymbol{\Omega}) .
\end{aligned}
$$

Using equations (7), (8), (14), and (15), we have

$$
\begin{aligned}
& \tilde{J}_{l m}(\boldsymbol{r})=(-1)^{l} J_{l m}(\boldsymbol{r}), \\
& \tilde{F}_{l m}(\boldsymbol{r})=(-1)^{l} F_{l m}(\boldsymbol{r}) .
\end{aligned}
$$

From equations (9), (13), (16), and (17) we can prove that $\tilde{J}$ is the solution of equation

$$
\left(1-K^{+}\right) \tilde{J}=\tilde{F}
$$

where

$$
\begin{aligned}
& \tilde{J}=\left[\begin{array}{llll}
\tilde{J}_{00}(\boldsymbol{r}), & \tilde{J}_{11}(\boldsymbol{r}), & \tilde{J}_{10}(\boldsymbol{r}), & \tilde{J}_{1-1}(\boldsymbol{r}), \cdots \tilde{J}_{l m}(\boldsymbol{r}), \cdots
\end{array}\right] \\
& \tilde{F}=\left[\begin{array}{llll}
\tilde{F}_{00}(\boldsymbol{r}), & \tilde{F}_{11}(\boldsymbol{r}), & \tilde{F}_{10}(\boldsymbol{r}), & \tilde{F}_{1-1}(\boldsymbol{r}), \cdots \tilde{F}_{l m}(\boldsymbol{r}), \cdots
\end{array}\right],
\end{aligned}
$$

and $K^{+}$is as defined in equation (13). Therefore equation (18) is nothing but equation (12) written in the new system! In summary, we have

$$
\begin{aligned}
(1-K) J_{\boldsymbol{k}_{0}} & =F_{\boldsymbol{k}_{0}}, \\
\left(1-K^{+}\right) \tilde{J}_{\boldsymbol{k}_{0}} & =\tilde{F}_{\boldsymbol{k}_{0}} .
\end{aligned}
$$

These equations are different expressions of the same physical process, and we have provided a simple physical interpretation of the adjoint operator $K^{+}$. Note that in going from equations (12) and (18) to equations (20) and (21) we have put in the explicit dependence of the parameter $\boldsymbol{k}_{0}$. With the help of the new coordinate system, we can express the differential cross section more concisely. Using equation (5) and substituting expressions (6) and (7) for $P(\boldsymbol{r} ; \boldsymbol{k}, \boldsymbol{\Omega})$ and $I(\boldsymbol{r}, \boldsymbol{\Omega})$, we have

$\frac{d \sigma}{d \Omega}\left(\boldsymbol{k}, \boldsymbol{k}_{0}\right)=\frac{1}{\pi F_{0}} \int_{0} d \boldsymbol{r} \sum_{l m} J_{l m}(\boldsymbol{r})\left[\frac{\alpha(\boldsymbol{r}) \omega_{l}(\boldsymbol{r})}{2 l+1}\right]^{1 / 2} \epsilon(\boldsymbol{r},-\boldsymbol{k}) Y_{l m}(\boldsymbol{k})$.

This can be further simplified using equation (10), and the final result is

$$
\frac{d \sigma}{d \Omega}\left(\boldsymbol{k}, \boldsymbol{k}_{0}\right)=\frac{1}{\pi^{2} F_{0}^{2}}\left\langle F_{-\boldsymbol{k}}, J_{\boldsymbol{k}_{0}}\right\rangle,
$$

where the scalar product is defined by

$$
\langle X, Y\rangle=\int_{0} d \boldsymbol{r} \sum_{l m} X_{l m}^{*}(\boldsymbol{r}) Y_{l m}(\boldsymbol{r})=\langle Y, X\rangle^{*}
$$

for any two vectors of the type as in equation (12). It is easy to show that $(d \sigma / d \Omega)\left(\boldsymbol{k}, \boldsymbol{k}_{0}\right)$ as given by equation (23) is real even though complex functions are involved.

It is of interest to consider the simultaneous solution of a forward scattering problem and its reverse. As shown in Figure $1 b$, in the forward problem the incident and emergent directions are $\boldsymbol{k}_{1}$ and $\boldsymbol{k}_{2}$, respectively. The corresponding directions in the reverse problem are $-\boldsymbol{k}_{2}$ and $-\boldsymbol{k}_{1}$, respectively. The appropriate field equations are

$$
\begin{aligned}
(1-K) J_{\boldsymbol{k}_{1}} & =F_{\boldsymbol{k}_{1}}, \\
\left(1-K^{+}\right) \tilde{J}_{-\boldsymbol{k}_{2}} & =\tilde{F}_{-\boldsymbol{k}_{2}} .
\end{aligned}
$$


The corresponding differential cross sections are

$$
\begin{aligned}
\frac{d \sigma}{d \Omega}\left(\boldsymbol{k}_{2}, \boldsymbol{k}_{1}\right) & =\frac{1}{\pi^{2} F_{0}^{2}}\left\langle F_{-\boldsymbol{k}_{2}}, J_{\boldsymbol{k}_{1}}\right\rangle \\
\frac{d \sigma}{d \Omega}\left(-\boldsymbol{k}_{1},-\boldsymbol{k}_{2}\right) & =\frac{1}{\pi^{2} F_{0}^{2}}\left\langle F_{\boldsymbol{k}_{1}}, J_{-\boldsymbol{k}_{2}}\right\rangle .
\end{aligned}
$$

Equations (25) and (26) can be written as a single equation in an extended double Hilbert space:

$$
L J=S,
$$

where

$$
\begin{aligned}
L & =\left[\begin{array}{cc}
0 & 1-K \\
1-K^{+} & 0
\end{array}\right], \\
J & =\left(\widetilde{J}_{-\boldsymbol{k}_{2}}, J_{\boldsymbol{k}_{1}}\right), \\
S & =\left(F_{\boldsymbol{k}_{1}}, \tilde{F}_{-\boldsymbol{k}_{2}}\right) .
\end{aligned}
$$

Since $L$ is a self-adjoint operator, we can construct a functional

$$
F(Q)=\langle Q, L Q\rangle-\langle Q, S\rangle-\langle S, Q\rangle,
$$

where $Q=(\tilde{q}, q)$ is an arbitrary vector in the double Hilbert space, and the scalar product is defined as the straightforward generalization of equation (24). On imposing the stationarity condition on equation (33) and using $L^{+}=L$, we have

$$
\delta F(Q)=\langle\delta Q, L Q-S\rangle-\langle L Q-S, \delta Q\rangle=0,
$$

which implies

$$
L Q=S .
$$

We recognize equation (35) as equivalent to equation (29) and, hence, equation (33) is the correct functional for the radiative transfer problem. We can use equation (33) and apply the usual variational techniques to obtain approximate solutions to equations (20) and (21). But there is an additional advantage, as we shall show in the following.

Let us evaluate equation (33) for some special choices of $Q$ : $Q_{0}=\left(\widetilde{J}_{-\boldsymbol{k}_{2}}, J_{\boldsymbol{k}_{1}}\right), Q_{1}=\left(\tilde{q}, J_{\boldsymbol{k}_{1}}\right)$, and $Q_{2}=\left(\widetilde{J}_{-\boldsymbol{k}_{2}}, q\right)$, where $\tilde{q}$ and $q$ are arbitrary, but $J_{\boldsymbol{k}_{1}}$ and $J_{-k_{2}}$ are the solution of equations (25) and (26), respectively. After some simple algebra, we have

$$
\begin{aligned}
F_{0} & =F\left(Q_{0}\right)=-\left\langle F_{\boldsymbol{k}_{1}}, \tilde{J}_{-\boldsymbol{k}_{2}}\right\rangle-\left\langle\tilde{F}_{-\boldsymbol{k}_{2}}, J_{\boldsymbol{k}_{1}}\right\rangle \\
& =-\left[\frac{d \sigma}{d \Omega}\left(-\boldsymbol{k}_{1},-\boldsymbol{k}_{2}\right)+\frac{d \sigma}{d \Omega}\left(\boldsymbol{k}_{2}, \boldsymbol{k}_{1}\right)\right] \pi^{2} F_{0}^{2}, \\
F_{1} & =F\left(Q_{1}\right)=-\left\langle J_{\boldsymbol{k}_{1}}, \tilde{F}_{-\boldsymbol{k}_{2}}\right\rangle-\left\langle\tilde{F}_{-\boldsymbol{k}_{2}}, J_{\boldsymbol{k}_{1}}\right\rangle \\
& =-2 \frac{d \sigma}{d \Omega}\left(\boldsymbol{k}_{2}, \boldsymbol{k}_{1}\right) \pi^{2} F_{0}^{2}, \\
F_{2} & =F\left(Q_{2}\right)=-\left\langle\tilde{J}_{-\boldsymbol{k}_{2}}, F_{\boldsymbol{k}_{1}}\right\rangle-\left\langle F_{\boldsymbol{k}_{1}}, \tilde{J}_{-\boldsymbol{k}_{2}}\right\rangle \\
& =-2 \frac{d \sigma}{d \Omega}\left(-\boldsymbol{k}_{1},-\boldsymbol{k}_{2}\right) \pi^{2} F_{0}^{2} .
\end{aligned}
$$

Since $\tilde{q}$ and $q$ are arbitrary, we must have

$$
\begin{aligned}
F_{0}=F_{1}=F_{2} & =-2 \pi^{2} F_{0}^{2} \frac{d \sigma}{d \Omega}\left(\boldsymbol{k}_{2}, \boldsymbol{k}_{1}\right) \\
& =-2 \pi^{2} F_{0}^{2} \frac{d \sigma}{d \Omega}\left(-\boldsymbol{k}_{1},-\boldsymbol{k}_{2}\right) .
\end{aligned}
$$

This proves that the differential cross section satisfies the principle of reciprocity (Minnaert 1941; Hovenier 1969) and that the extremum of the functional (33) is proportional to the differential cross section.

The results summarized in equations (33) and (39) are new and provide a generalization of previous work on solving the planetary problem in radiative transfer using variational methods (Huang 1953; Stokes and DeMarcus 1971; Cheyney and Arking 1976; Sze 1976; Yung 1976; Chow, Friedson, and Yung 1984). Our work reveals the interconnection of the microscopic symmetry of the phase function $P\left(\boldsymbol{r} ; \boldsymbol{\Omega}_{1}, \boldsymbol{\Omega}_{2}\right)$, the macroscopic symmetry of the differential cross section $(d \sigma / d \Omega)$ $\left(\boldsymbol{k}_{2}, \boldsymbol{k}_{1}\right)$, and the variational principle. Actually, the symmetry given by equation (6) is unnecessarily strong. A weaker microscopic symmetry $P\left(\boldsymbol{r} ; \boldsymbol{\Omega}_{1}, \boldsymbol{\Omega}_{2}\right)=P\left(\boldsymbol{r} ;-\boldsymbol{\Omega}_{2},-\boldsymbol{\Omega}_{1}\right)$ is sufficient to ensure the macroscopic symmetry $(d \sigma / d \Omega)\left(\boldsymbol{k}_{2}, \boldsymbol{k}_{1}\right)=(d \sigma / d \Omega)$ $\left(-\boldsymbol{k}_{1},-\boldsymbol{k}_{2}\right)$ (Case 1957), and the existence of a variational principle. In Appendix $\mathrm{C}$ we give an alternative proof of our results using the weaker microscopic symmetry assumption. We also show that if we use the method of successive orders to compute the radiation field to order $n$, then the differential cross section evaluated using equation (33) is accurate to order $2 n$. The usefulness of symmetry is appreciated by van de Hulst (1980a, p. 17): “Such symmetry relations are of great practica! help in checking the consistency of analytic formulae or of computational results." But he did not recognize the full potential of such relations. The recognition of the importance of variational methods in radiative transfer is overdue, especially in light of notable advances in using variational methods in related fields such as neutron transport (Francis et al. 1959; Pomraning and Clark 1963a,b), kinetic theory (Cercignani and Pagani 1966; Cercignani 1969), electromagnetic wave scattering (Levine and Schwinger 1950), acoustic wave scattering (Gerjuoy and Saxon 1954), and quantum theory of scattering (Lippmann and Schwinger 1950). Viewed in the broader context, our results are not only reasonable, they are inevitable (Gerjuoy, Rau, and Spruch 1983).

\section{HOMOGENEOUS SPHERE WITH ISOTROPIC SCATTERERS}

We will now apply the integral equation to the particularly simple case of a homogeneous sphere of radius $a$ filled with isotropic scatterers of single scattering albedo $\omega_{0}$. In this case, only the $l=0$ equation is needed. From equation (9) we have

$$
J_{00}\left(\boldsymbol{r}_{1}\right)=\frac{\alpha \omega_{0}}{4 \pi} \int d \boldsymbol{r}_{2} \frac{e^{-\tau\left(\boldsymbol{r}_{1}, \boldsymbol{r}_{2}\right)}}{r_{12}^{2}} J_{00}\left(\boldsymbol{r}_{2}\right)+F_{00}\left(\boldsymbol{r}_{1}\right) .
$$

Since the medium is homogeneous, we can replace the distance variable by an optical variable

$$
\tau=\alpha \boldsymbol{r} \text {. }
$$

Note that $\tau_{0}=\alpha a$ and $\tau_{12}=\tau\left(\boldsymbol{r}_{1}, \boldsymbol{r}_{2}\right)=\alpha\left|\boldsymbol{r}_{1}-\boldsymbol{r}_{2}\right|$. Then equation (40) becomes

$$
J\left(\tau_{1}\right)=J_{0}\left(\tau_{1}\right)+\omega_{0} \int d \tau_{2} G\left(\tau_{1}, \tau_{2}\right) J\left(\tau_{2}\right),
$$

where the mean intensity is

$$
J(\tau)=\frac{1}{4 \pi} \int \mathrm{d} \boldsymbol{\Omega} I(\tau, \boldsymbol{\Omega})=\left[\frac{1}{4 \pi \alpha \omega_{0}}\right]^{1 / 2} J_{00}(\tau),
$$

and $J(\tau)$ is not the same as the $J$ in equation (12) or $J_{00}(\tau)$ in 
equation (40); the solar term is

$$
\begin{aligned}
J_{0}(\tau) & =\frac{1}{4 \pi} \int d \boldsymbol{\Omega} F(\tau, \boldsymbol{\Omega})=\frac{F_{0}}{4} \epsilon\left(\tau, \boldsymbol{k}_{0}\right) \\
& =\frac{F_{0}}{4} e^{-\left[\tau_{0}-\tau^{2}+\left(\tau \cdot k_{0}\right)^{2}\right]^{1 / 2} / \tau \cdot k_{0}},
\end{aligned}
$$

and Green's function of the integral equation is

$$
G\left(\tau_{1}, \tau_{2}\right)=\frac{e^{-\tau_{12}}}{4 \pi \tau_{12}^{2}}
$$

The integration of the $\tau_{2}$ variable is over the entire optical sphere of radius $\tau_{0}$. This equation is known in the literature as the Peierls equation (Peierls 1939).

Equation (41) bears striking similarity to the integral equation that describes the scattering of a scalar wave of wavenumber $\boldsymbol{k}$ by a dielectric sphere with index of refraction equal to $m$ (Jackson 1975, pp. 739-743):

$$
\psi\left(\boldsymbol{r}_{1}\right)=e^{i \boldsymbol{k} \cdot \boldsymbol{r}_{1}}+k^{2}\left(m^{2}-1\right) \int d \boldsymbol{r}_{2} G^{w}\left(\boldsymbol{r}_{1}, \boldsymbol{r}_{2} ; k\right) \psi\left(\boldsymbol{r}_{2}\right),
$$

where Green's function is

$$
\begin{aligned}
G^{w}\left(\boldsymbol{r}_{1}, \boldsymbol{r}_{2} ; k\right)= & \frac{e^{i k r_{12}}}{4 \pi r_{12}} \\
= & i k \sum_{l=0}^{\infty}(2 l+1) j_{l}\left(k r_{<}\right) h_{l}^{(1)}\left(k r_{>}\right) \\
& \times \sum_{m=-l}^{l} Y_{l m}^{*}\left(\vartheta_{1}, \varphi_{1}\right) Y_{l m}\left(\vartheta_{2}, \varphi_{2}\right),
\end{aligned}
$$

and $r_{<}=\min \left(r_{1}, r_{2}\right), r_{>}=\max \left(r_{1}, r_{2}\right)$, and $j_{l}$ and $h_{l}^{(1)}$ are spherical Bessel functions of the first and the third kind, respectively. Note that

$$
G\left(\tau_{1}, \tau_{2}\right)=\int_{1}^{\infty} d \sigma G^{w}\left(\tau_{1}, \tau_{2} ; i \sigma\right)
$$

This suggests a close connection between equations (41) and (45), and that the multipole expansion method first developed for solving equation (45) would be very useful for solving equation (41). Because of the cylindrical symmetry around the Sunsphere axis (see Fig. $10 b$ ) we can expand $J(\tau)$ and $J_{0}(\tau)$, using the Legendre polynomials:

$$
\begin{gathered}
J(\tau)=J(\tau, \mu)=\sum_{l=0}^{\infty} \tau^{-1} \psi_{l}(\tau) P_{l}(\mu), \\
J_{0}(\tau)=J_{0}(\tau, \mu)=\sum_{l=0}^{\infty} \tau^{-1} S_{l}(\tau) P_{l}(\mu),
\end{gathered}
$$

where $\tau=|\tau|$ and $\mu=\boldsymbol{k}_{0} \cdot \tau / \tau$ is the cosine of the angle between the direction of incident radiation $\boldsymbol{k}_{0}$ and the position vector $\tau$. Substituting equations (45), (46), (47), and (48) into equation (41) and integrating with respect to $\varphi_{1}$ and $\varphi_{2}$, we get a set of uncoupled integral equations for $l=0,1,2 \ldots$,

$$
\psi_{l}\left(\tau_{1}\right)=S_{l}\left(\tau_{1}\right)+\omega_{0} \int_{0}^{\tau_{0}} d \tau_{2} G_{l}\left(\tau_{1}, \tau_{2}\right) \psi_{l}\left(\tau_{2}\right)
$$

where

$$
\begin{gathered}
G_{l}\left(\tau_{1}, \tau_{2}\right)=-\tau_{1} \tau_{2} \int_{1}^{\infty} d \sigma \sigma j_{l}\left(i \sigma \tau_{<}\right) h_{l}^{(1)}\left(i \sigma \tau_{>}\right) \\
=\sum_{m=0}^{2 l} \frac{1}{2}\left[(-1)^{l+1} A_{l m} E_{m+1}\left(\tau_{>}+\tau_{<}\right)\right. \\
\left.\quad+B_{l m} E_{m+1}\left(\tau_{>}-\tau_{<}\right)\right]
\end{gathered}
$$

with

$$
\begin{gathered}
A_{l m}=\sum_{k=0}^{l} \sum_{k^{\prime}=0}^{l} \delta_{k+k^{\prime}, m} \frac{(l+k) !\left(l+k^{\prime}\right) !}{2^{m} \tau_{<}^{k} \tau_{>}^{k^{\prime}} k ! k^{\prime} !(l-k) !\left(l-k^{\prime}\right) !}, \\
B_{l m}=\sum_{k=0}^{l} \sum_{k^{\prime}=0}^{l} \delta_{k+k^{\prime}, m} \frac{(-1)^{k}(l+k) !\left(l+k^{\prime}\right) !}{2^{m} \tau_{<}^{k} \tau_{>}^{k^{\prime}} k ! k^{\prime} !(l-k) !\left(l-k^{\prime}\right) !}, \\
E_{n}(x) \equiv \int_{1}^{\infty} \frac{e^{-x t}}{t^{n}} d t .
\end{gathered}
$$

A detailed derivation of equation (50) and an examination of the properties of Green's function are referred to Appendix D. To gain insight into the meaning of equation (50), let us investigate the special case $l=0$ :

$$
\begin{aligned}
\psi_{0}\left(\tau_{1}\right)= & S_{0}\left(\tau_{1}\right)+\frac{1}{2} \omega_{0} \\
& \times \int_{0}^{\tau_{0}} d \tau_{2}\left[E_{1}\left(\left|\tau_{1}-\tau_{2}\right|\right)-E_{1}\left(\tau_{1}+\tau_{2}\right)\right] \psi_{0}\left(\tau_{2}\right) .
\end{aligned}
$$

This is the same as equation (3) in Sobolev (1972), and is the Milne integral equation for a homogeneous sphere (see Appendix of Sobolev 1975). However, to solve the complete planetary problem with the source of illumination placed at infinity we need the entire series (48). Thus, our results are a generalization of Sobolev (1972). In practice we have to truncate equation (48) at some finite $l=l_{\max }$, and the series is useful only if it converges rapidly. That this is so will be discussed in the following section.

The differential cross section, according to equation (5), is

$$
\frac{d \sigma}{d \Omega}\left(\boldsymbol{k}, \boldsymbol{k}_{0}\right)=\frac{\omega_{0}}{\pi F_{0} \alpha^{2}} \int d \tau \epsilon(\tau,-\boldsymbol{k}) J(\tau) .
$$

But the expression can be further simplified using equations (48), (49), and Appendix B:

$$
\frac{d \sigma}{d \Omega}\left(\alpha_{s}\right)=\frac{\omega_{0}}{\alpha^{2}} \sum_{l=0}^{\infty}(-1)^{l} f_{l} P_{l}\left(\cos \alpha_{s}\right),
$$

with

$$
f_{l}=\frac{16}{F_{0}^{2}} \frac{1}{2 l+1} \int_{0}^{\tau_{0}} d \tau S_{l}(\tau) \psi_{l}(\tau)
$$

and $\alpha_{s}$ is the scattering angle defined by $\cos \alpha_{s}=\boldsymbol{k} \cdot \boldsymbol{k}_{0}$ (see Fig. 10b). There is a host of interesting functions that can be given in terms of the differential cross section. Two distinct sets of nomenclature exist, depending on whether the overall scattering is viewed as due to a particle or a planet. In the former case we are interested in the extinction and absorption cross section, the $g$-factor, and the scattering phase function. In the latter case, the relevant quantities are the geometric albedo, the phase variation, the phase integral, and the bond albedo. Appendix A provides a listing of the important "particle" and "planetary" photometric functions taken from standard references (van de Hulst 1957; Horak 1950; Harris 1961) and their evolution in terms of the multipole functions (54) and (55).

\section{NUMERICAL RESULTS AND DISCUSSION}

In our numerical model we evaluate the solar term (49) using a 36 point Gaussian quadrature. Green's function (eq. [51]) is computed using a procedure described in Appendix D. The integral equation (50) is discretized, with $100-200$ points between 0 and $\tau_{0}$, and the resulting matrix equation is solved 
as in Sze (1976) and Yung (1976). A large number of cases have been studied for $\tau_{0}$ in the range $0-8$ and for $\omega_{0}$ from 0 to 1 . For the requirement of an overall accuracy of order $3 \%$, it turns out that the maximum number of multipole equations, $l_{\max }$ +1 , is less than 12 . We will present detailed results for two representative cases, $\tau_{0}=1$ and $\tau_{0}=8$, both with $\omega_{0}=1$. These results will serve to illustrate the character of our solutions for spheres of small to moderate optical thickness. A large amount of interesting information deduced from our numerical studies is summarized in the graphs of photometric functions.

The multipole expansions of the solar term $S_{l}(\tau)$ are shown in Figures $2 a$ and $2 b$ for the cases $\tau_{0}=1$ and 8 , respectively. Note that the higher multipoles decrease rapidly as $l$ approaches and exceeds $\tau_{0}$. Figures $3 a$ and $3 b$ show the corresponding multipole solutions $\psi_{l}(\tau)$. The solutions display similar properties as the solar terms. The mean intensity of the internal radiation field (as defined by eq. [48]) on the Sunsphere axis $J(\tau,-1)$ and its comparison with the primary solar term $J_{0}(\tau,-1)$ are shown in Figure $4 a$ for $\tau_{0}=1$. Similar results for the case $\tau_{0}=8$ are presented in Figure $4 b$. The comparison clearly demonstrates the importance of multiple scattering for determining the internal radiation field inside a sphere. Table 1 provides a summary of the integrals of the multipole radiation fields $f_{l}$ for $\tau_{0}=1$ and 8 . This table gives a proper assessment of the relative contribution of the higher multipoles.

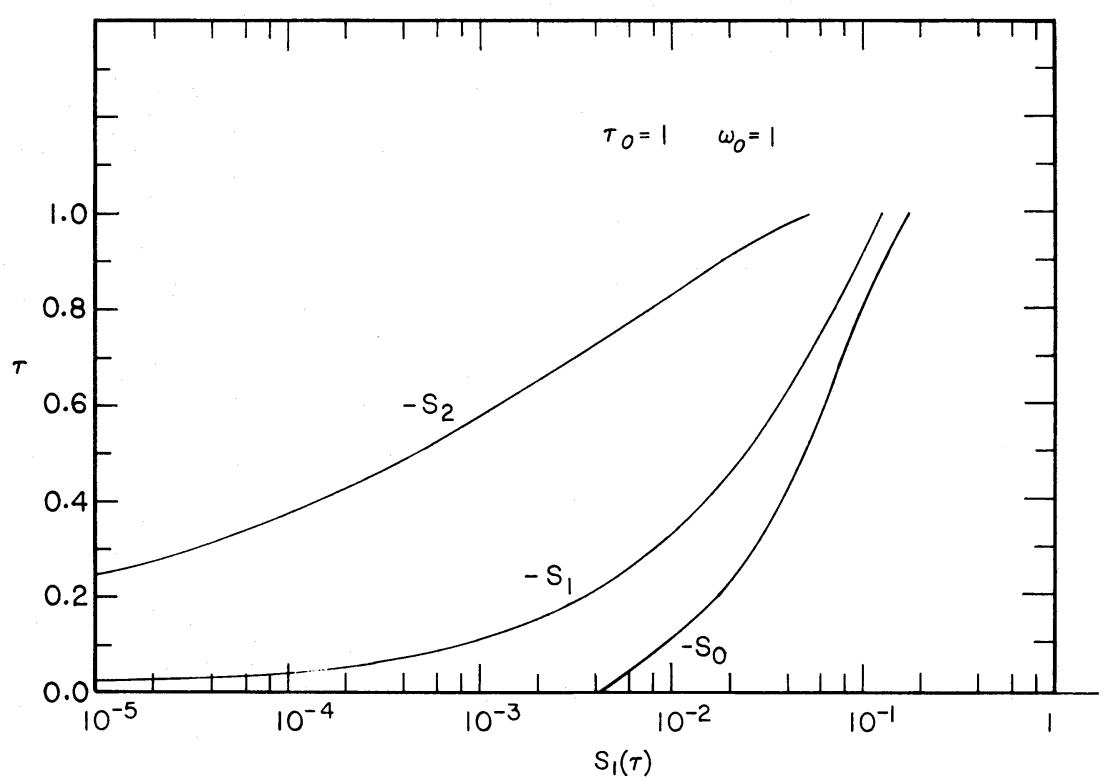

FIG. $2 a$

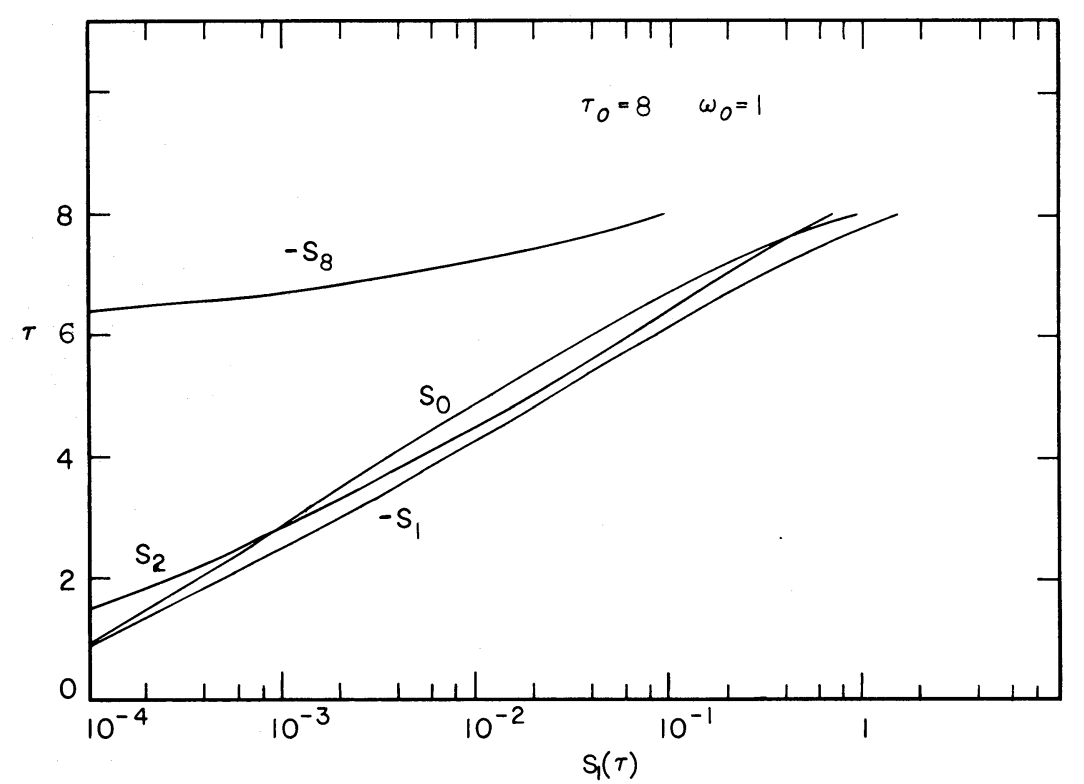

FIG. $2 b$

FIG. 2.-Source function in eq. (50). (a) $S_{0}, S_{1}$, and $S_{2}$ for $\tau_{0}=1$ case. (b) $S_{0}, S_{1}, S_{2}, S_{8}$ for $\tau_{0}=8$ case. 


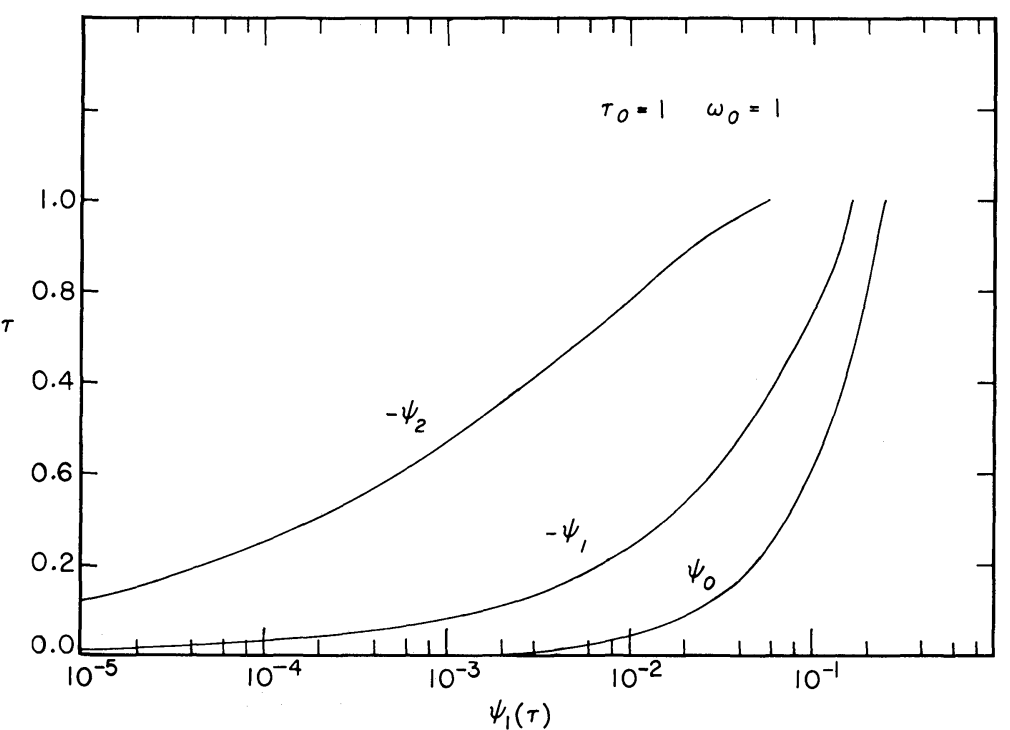

Fig. $3 a$

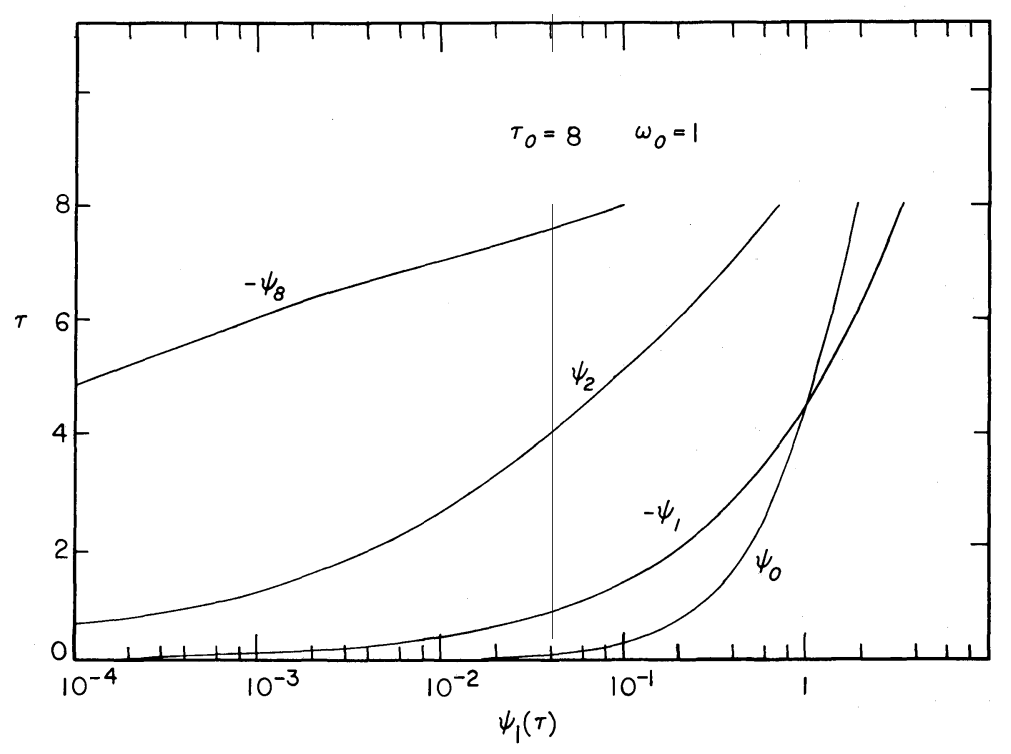

FIG. $3 b$

FIG. 3.-Solution of eq. (50), (a) $\psi_{0}, \psi_{1}, \psi_{2}$ for $\tau_{0}=1$ case. (b) $\psi_{0}, \psi_{1}, \psi_{2}, \psi_{8}$ for $\tau_{0}=8$ case.
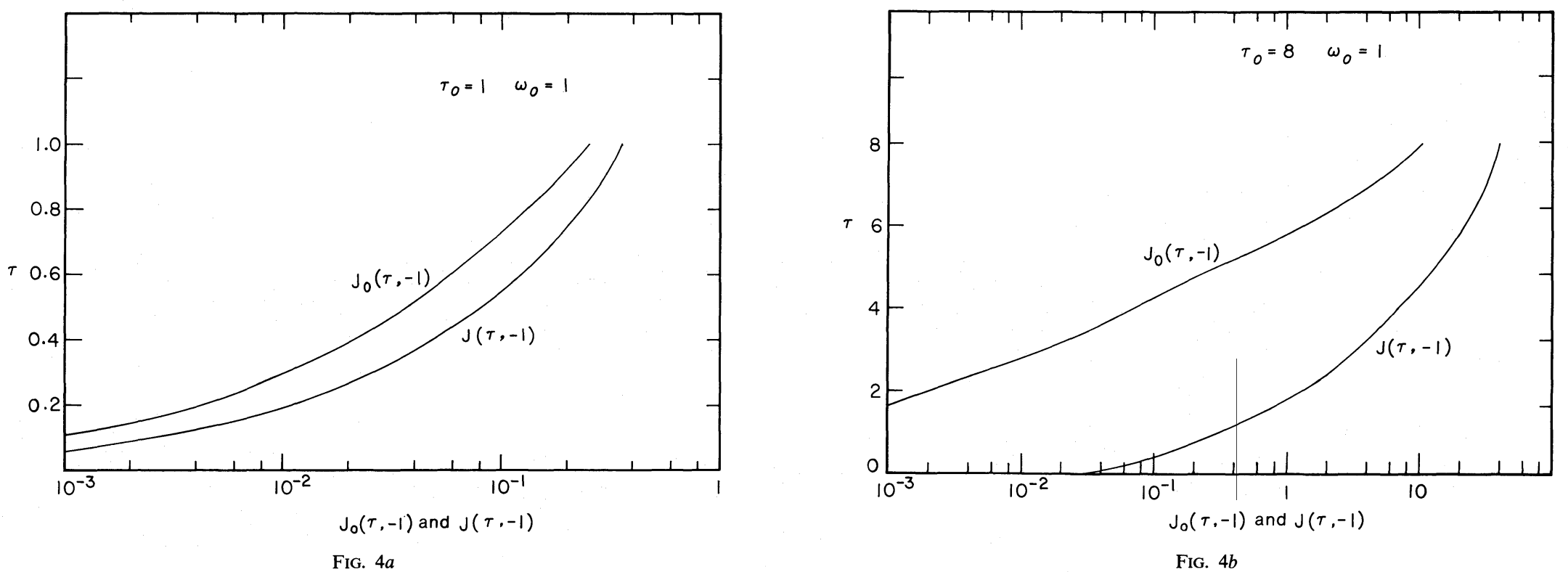

FIG. $4 b$

FIG. 4.-Internal mean intensity of radiation as a function of $\tau$. (a) $J_{0}(\tau, \mu=-1), J(\tau, \mu=-1)$ for $\tau_{0}=1$ case. $(b) J_{0}(\tau, \mu=-1), J(\tau, \mu=-1)$ for $\tau_{0}=8$ case. 
TABLE 1

VALUES OF $f_{l}$ FOR $\tau_{0}=1$ AND $8^{\text {a }}$

\begin{tabular}{|c|c|c|}
\hline$l$ & $f_{l}\left(\tau_{0}=1\right)$ & $f_{l}\left(\tau_{0}=8\right)$ \\
\hline $0 \ldots \ldots \ldots$ & $4.39 \times 10^{-2}$ & 3.49 \\
\hline $1 \ldots \ldots \ldots \ldots$ & $5.48 \times 10^{-3}$ & 3.22 \\
\hline $2 \ldots \ldots$ & $1.30 \times 10^{-4}$ & 0.12 \\
\hline $3 \ldots \ldots$ & $4.8 \times 10^{-5}$ & 0.05 \\
\hline $4 \ldots \ldots \ldots \ldots$ & $1.4 \times 10^{-5}$ & $9.4 \times 10^{-3}$ \\
\hline $5 \ldots \ldots \ldots \ldots$ & $2.0 \times 10^{-6}$ & $1.1 \times 10^{-2}$ \\
\hline $6 \ldots \ldots$ & $2.1 \times 10^{-6}$ & $1.5 \times 10^{-3}$ \\
\hline $7 \ldots \ldots \ldots$ & $1.9 \times 10^{-7}$ & $3.5 \times 10^{-3}$ \\
\hline $8 \ldots \ldots \ldots \ldots$ & $4.5 \times 10^{-7}$ & $3.3 \times 10^{-4}$ \\
\hline $9 \ldots \ldots \ldots \ldots$ & $2.9 \times 10^{-8}$ & $1.4 \times 10^{-4}$ \\
\hline $10 \ldots \ldots \ldots \ldots$ & $1.3 \times 10^{-7}$ & $9.6 \times 10^{-5}$ \\
\hline
\end{tabular}

a See eq. (55) for definition of $f_{l}$.

Values for the extinction efficiency $Q_{\text {ext }}$ are presented in Figure 5. This is a measure of the efficiency of the sphere for absorbing incident light relative to a disk of area $\pi a^{2}$. The limiting values can be easily shown to be

$$
\lim _{\tau_{0} \rightarrow 0} Q_{\text {ext }}=\frac{4}{3} \tau_{0}, \quad \lim _{\tau_{0} \rightarrow \infty} Q_{\text {ext }}=1 .
$$

We note that as $\tau_{0}$ exceeds $8, Q_{\text {ext }}$ exceeds $99 \%$, and, hence, the sphere is essentially opaque. The scattering efficiency $Q_{\text {sca }}$ is shown as a function of $\omega_{0}$ in Figure 6 . The quantity $Q_{\text {sca }}$, by definition, is the same as the Bond albedo. The case $\tau_{0}=\infty$ is taken from Irvine's (1975) Figure 5 for the Bond albedo (or spherical albedo). By conservation of energy, $Q_{\text {sca }}=Q_{\text {ext }}$ for $\omega_{0}=1$. The limiting values of $Q_{\text {sca }}$ are (van de Hulst $1980 b, \mathrm{p}$. 369)

$$
\begin{aligned}
& \lim _{\tau_{0} \rightarrow \infty} Q_{\text {sca }} \approx(1-s) \frac{1-0.139 s}{1+1.170 s}, \\
& \lim _{\tau_{0} \rightarrow 0} Q_{\text {sca }}=\frac{4}{3} \omega_{0} \tau_{0},
\end{aligned}
$$

where $s=\left(1-\omega_{0}\right)^{1 / 2}$. The first expression reflects the importance of multiple scattering, as is obvious from the appearance of the factor $1-\left(1-\omega_{0}\right)^{1 / 2}$. (For an illuminating discussion of this factor the reader is referred to McElroy 1971.) The second expression includes only single scattering. The asymmetry factor $g$ is shown in Figure 7. For a sphere with isotropic scatterers, $g$ is always negative. The limiting values of $g$ are

$$
\lim _{\tau_{0} \rightarrow \infty} g=-0.4473, \quad \lim _{\tau_{0} \rightarrow 0} g=0 .
$$

The large sphere value is approximately computed using the tabulation of Harris (1961) and should be compared with $g=$ $-\frac{4}{9}$ for a Lambert sphere (van de Hulst 1980b, p. 606).

Figure 8 shows values of the geometric albedo of a sphere as a function of $\omega_{0}$ for a range of values of $\tau_{0}$. The case of $\tau_{0}=\infty$ is taken from Harris (1961). In the small sphere limit we have

$$
\lim _{\tau_{0} \rightarrow 0} p=\frac{1}{3} \omega_{0} \tau_{0} \text {. }
$$

The values of the phase integral $q$ can be deduced from the relation

$$
p q=A=Q_{\text {sca }}
$$

and are not separately plotted. The phase variation $\varphi\left(\alpha_{p}\right)$ as a function of the phase angle $\alpha_{p}$ is shown in Figure 9 for $\tau_{0}=1$, 8 , and $\infty$ with $\omega_{0}=1$. For comparison we also present the case of a Lambert sphere. It is clear that as $\tau_{0}$ increases the scattering becomes more backward peaked and the phase variation approaches that of a Lambert sphere (van de Hulst $1980 b$, p. 606):

$$
\varphi\left(\alpha_{p}\right)=\frac{1}{\pi}\left[\left(\pi-\alpha_{p}\right) \cos \alpha_{p}+\sin \alpha_{p}\right] .
$$

Note that $\varphi\left(\alpha_{p}\right)$ is related to the scattering phase function $p\left(\alpha_{s}\right)$ by a normalization constant and a change of variable.

\section{APPLICATIONS}

The results we have presented in this initial report tend to emphasize the theoretical rather than the practical aspects of our work. More realistic modeling is not difficult in principle. However, before we build more complicated models we will discuss qualitatively the possible applications of our results to a number of interesting problems.

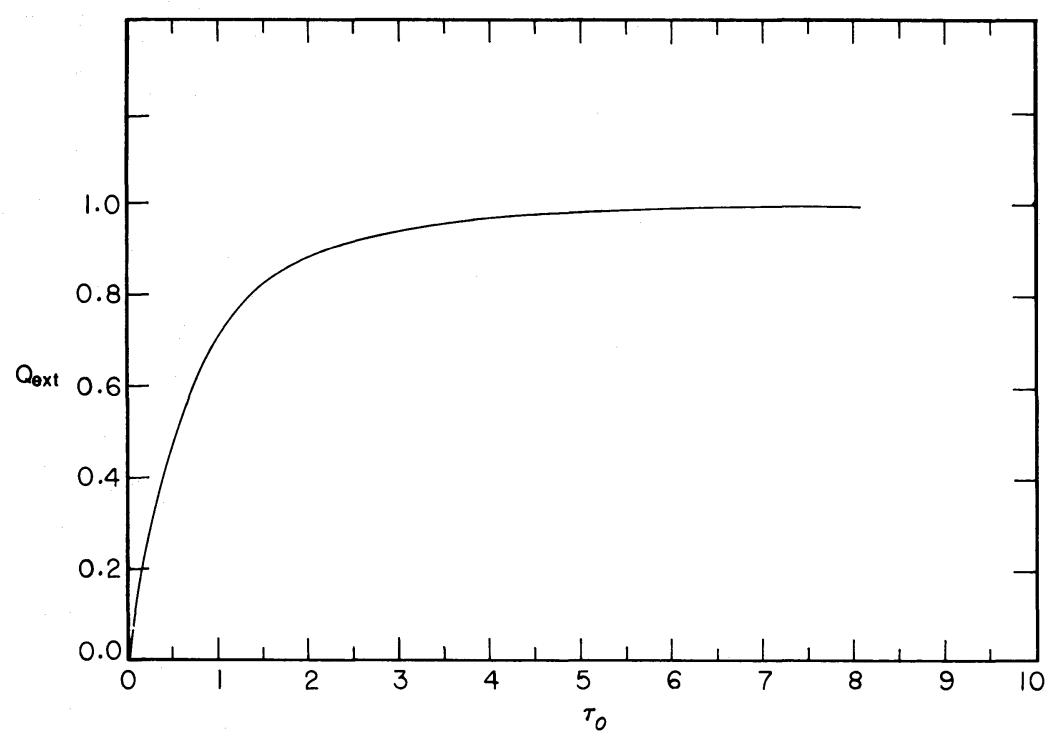

FIG. 5.-Extinction efficiency $Q_{\text {ext }}$ as a function of $\tau$ for $\tau=1-8$ 


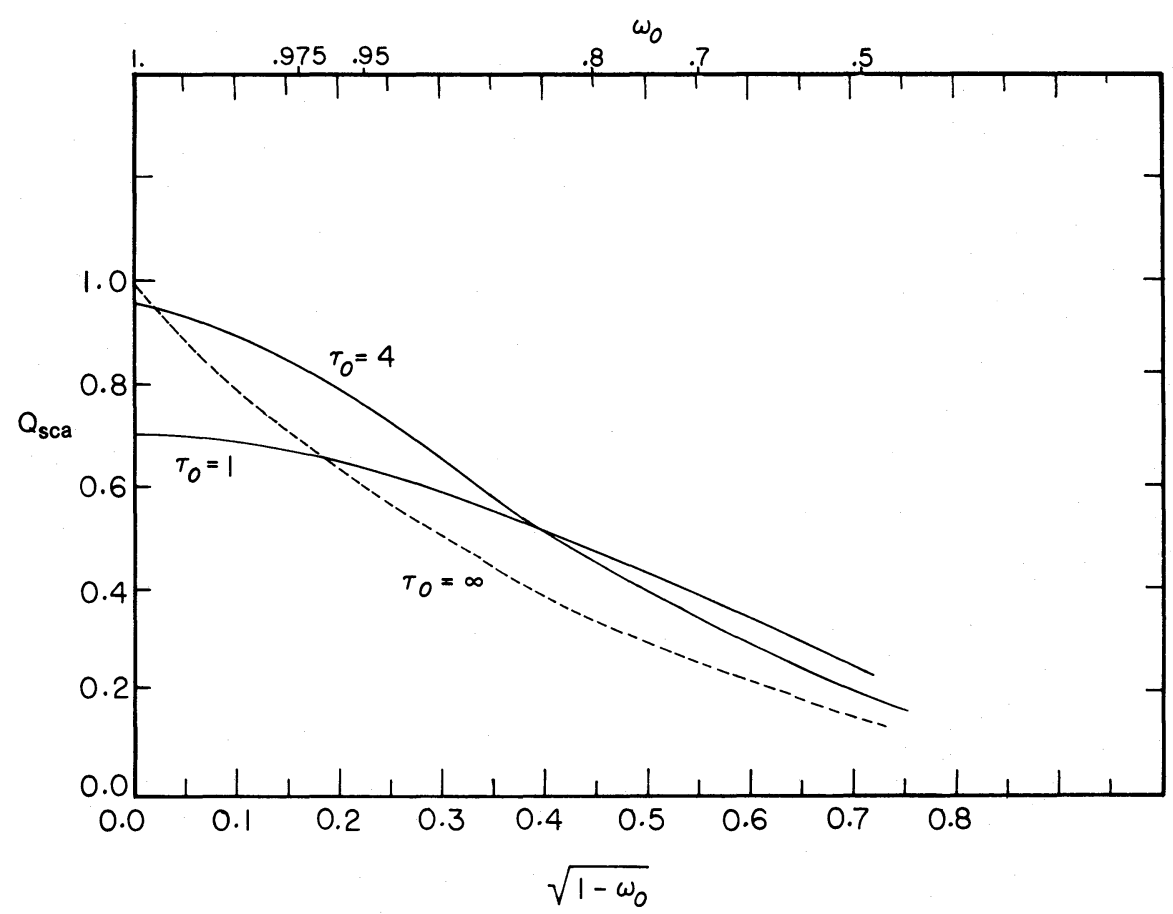

Fig. 6. - Scattering efficiency $Q_{\text {sca }}$ as a function of $\omega_{0}$ for $\tau_{0}=1$ and $\tau_{0}=4$ cases. The case $\tau_{0}=\infty$ is taken from Irvine's (1975) Fig. 5.

The photometry of Jupiter has been investigated by Cochran (1977), Tomasko, West, and Castillo (1978), Sato and Hansen (1979), and Smith and Tomasko (1984), and there is general agreement that the cloud phase function can be represented by a two-term Henyey-Greenstein phase function,

$$
P\left(\alpha_{s}\right)=f_{1} P\left(g_{1}, \alpha_{s}\right)+\left(1-f_{1}\right) P\left(g_{2}, \alpha_{s}\right),
$$

with

$$
P\left(g, \alpha_{s}\right)=\frac{1-g^{2}}{\left(1+g^{2}-2 g \cos \alpha_{s}\right)^{3 / 2}},
$$

where $\alpha_{s}$ is the scattering angle and $g$ is the asymmetry par- ameter. The best choices of the parameters are $f_{1}=0.938$, $g_{1}=0.80$, and $g_{2}=-0.65$. The first part of the phase function with forward-scattering $g_{1}=0.80$ poses no problem, since this is typical of most cloud particles (Hansen 1969). The origin of the second part of the phase function with backward scattering is obscure. The phase function of ammonia crystals, believed to be what the cloud particles are composed of, do not exhibit a pronounced backward peak (Tomasko and Doose 1984). We propose an explanation. Figure 7 shows that the asymmetry factor for patches of thick spherical clouds is always negative. In fact, if the clouds of Jupiter were not horizontally homogeneous, but possess small-scale structures, negative values of $g$ would be inevitable. But, of course, to provide a realistic inter-

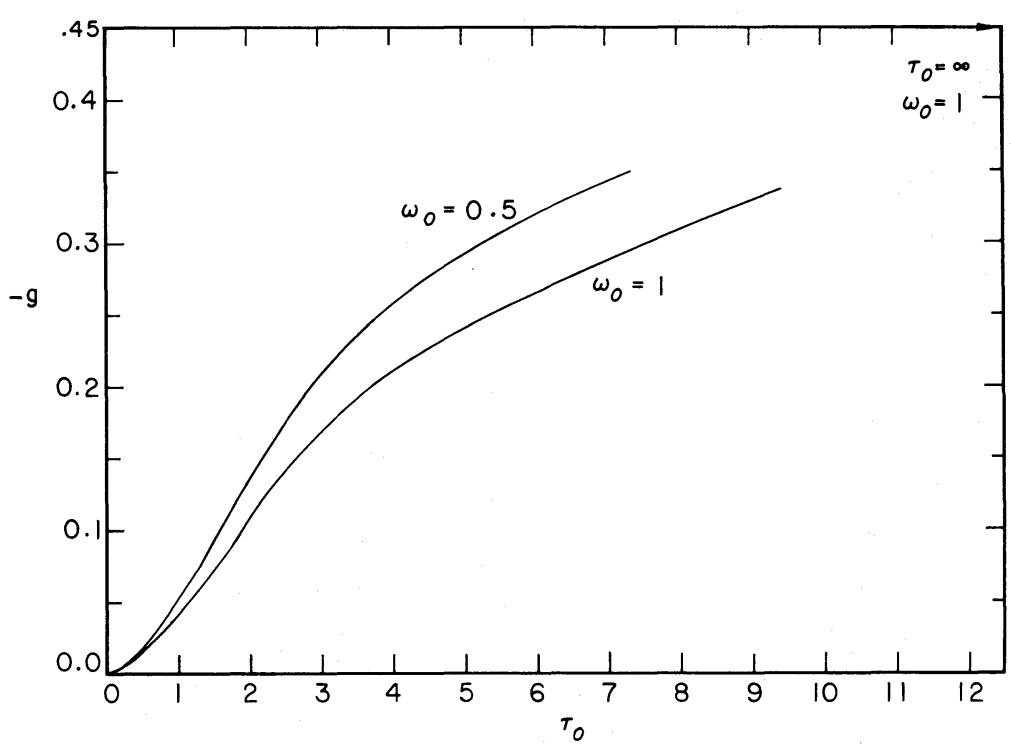

FIG. 7.-Asymmetry factor $g$ as a function of $\tau$ for $\omega_{0}=1$ and $\omega_{0}=0.5$ cases 


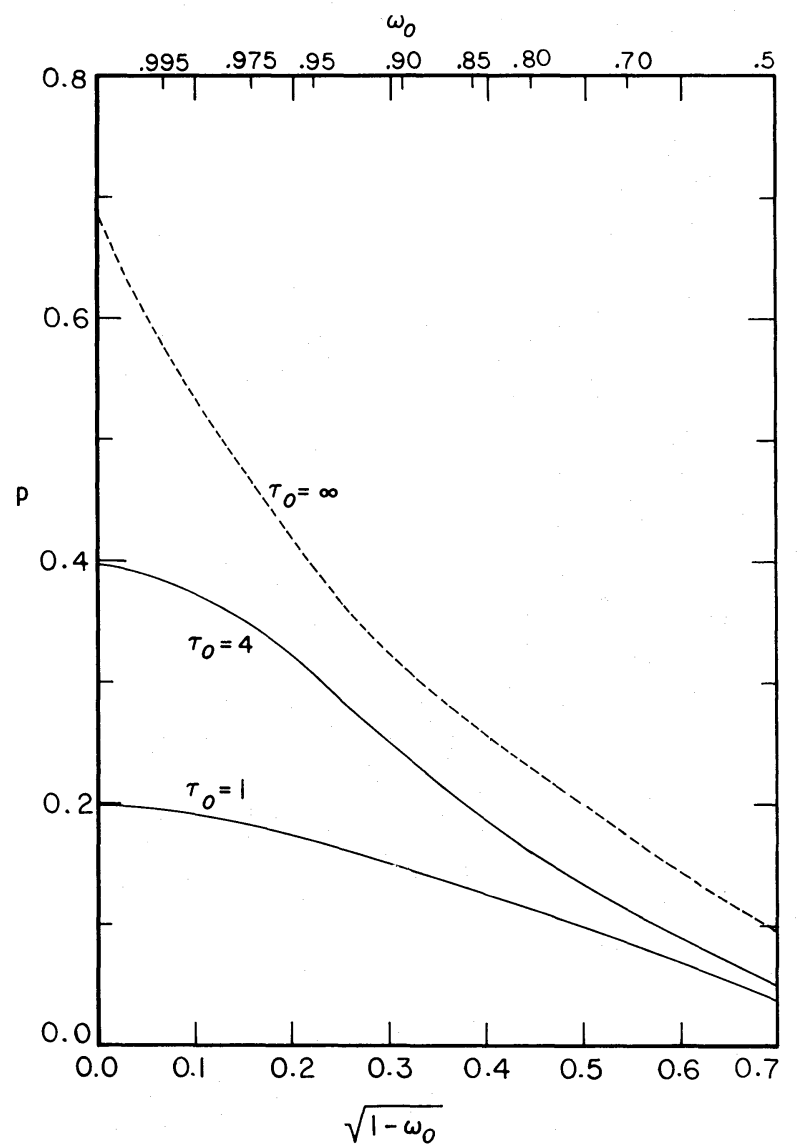

Fig. 8.-Geometric albedo of a sphere $p$ as a function of $\omega_{0}$ for $\tau_{0}=1$ and $\tau_{0}=4$ cases. The case of $\tau_{0}=\infty$ is taken from Harris (1961).

pretation of the photometry of Jupiter we have to make our spherical clouds out of anisotropic scatterers. This quantitative modeling must be deferred to the future.

We can apply our method to the study of planetary coronae arising from resonance scattering of solar photons (Modali, Brandt, and Kastner 1975; Anderson and Hord 1977) and the radiation field in the terrestrial stratosphere and troposphere at twilight. However, a complication arises in these problems due to the presence of the solid planetary body, which is opaque to radiation. In this case, equation (1) must be modified so that it becomes the appropriate equation of radiative transfer in the spherical shell atmosphere.

In a cometary atmosphere the geometry is roughly spherical. The scattering of sunlight in the continuum is dominated by micron-sized dust grains with low single scattering albedo $\omega_{0} \approx 0.1-0.4$ (Ney and Merrill 1976; Hanner 1979). The total optical depth is at most of order a few (Hellmich 1981), since greater optical depths would shield the incident sunlight from reaching the nucleus and cut off the production of gas and dust. In this problem, the best approach would be to use equation (1) and apply the method of successive orders and the variational principle (Appendix $C$ ). However, $I(\boldsymbol{r}, \boldsymbol{\Omega})$ is a function of five variables, and, therefore, solving equation (1) by any numerical scheme is a formidable task. In this case, it is desirable to find a transformation (such as eq. [48]) that would allow us to separate at least one or two independent variables using the intrinsic symmetry properties of the sphere, the scattering geometry, and the phase function.

The method developed in $\S$ IV is useful as a first approximation. The scattering phase function of the dust grains in a comet is not isotropic, but can be approximated by

$$
P\left(\boldsymbol{r} ; \mathbf{\Omega}_{1}, \mathbf{\Omega}_{2}\right)=\frac{1}{4 \pi}\left[4 \pi f \delta\left(\mathbf{\Omega}_{1}-\mathbf{\Omega}_{2}\right)+(1-f)\right],
$$

where $0 \leq f \leq 1$. In this case as in the case of a plane-parallel atmosphere (Sobolev 1975, $\S 8.3$ ), the problem can be reduced to the isotropic one using a suitable transformation.

\section{CONCLUSION}

In the past $30 \mathrm{yr}$ the theory of radiative transfer in slab geometry has reached the level of maturity comparable to other branches of mathematical physics (Chandrasekhar 1960; van de Hulst $1980 a, b$ ). However, the theory is still primitive for other geometries of practical interest. The integral equation approach holds great potential for advancing the subject. This method has at least three advantages: $(a)$ there are no bound-

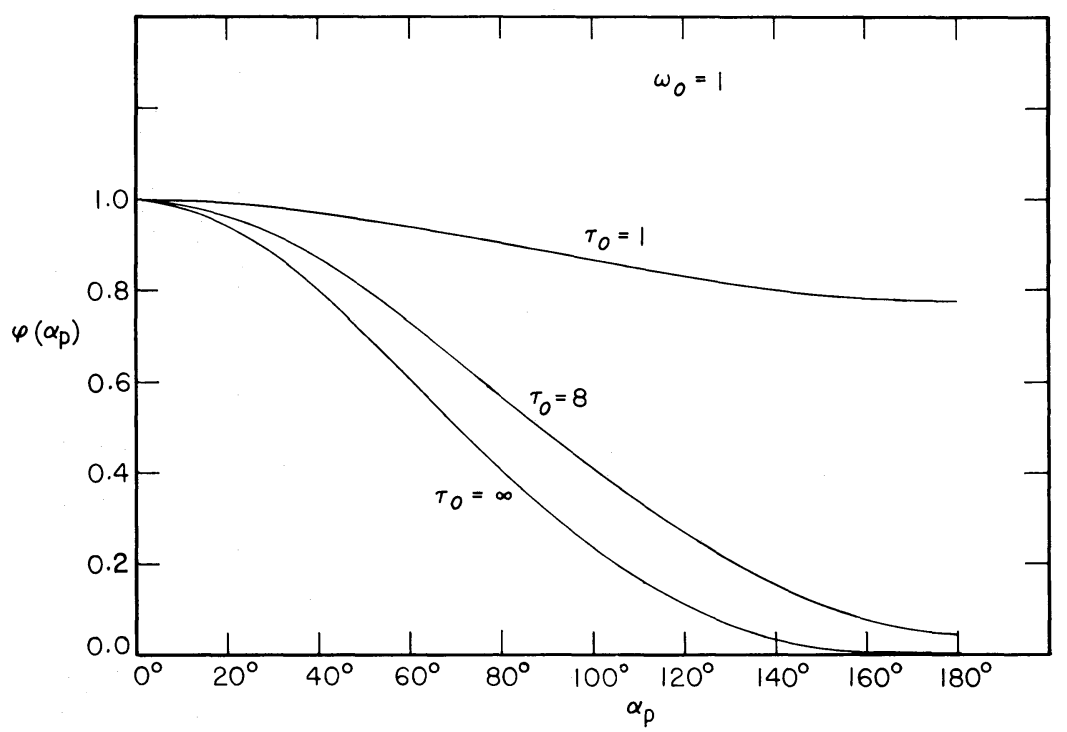

FIG. 9.-Phase variation $\varphi\left(\alpha_{p}\right)$ as a function of phase angle $\alpha_{p}$ for $\tau_{0}=1$ and $\tau_{0}=8$ cases. The case of $\tau_{0}=\infty$ is taken from van de Hulst (1980). 
ary conditions, and the integral equation contains the complete formulation of the radiative transfer problem; $(b)$ the important photometric quantity, the differential cross section, can be computed using a functional expression that is highly accurate for reasonably accurate radiation fields; $(c)$ in many cases the symmetry of the equation is such as to suggest a simple transformation that results in the separation of variables for the radiation field.

In this paper we explore and exploit some of the beauties and subtleties of the integral equation. A complete variational formulation of the radiative transfer of nonpolarized light is given in $\S$ III. The derivation reveals a profound connection, hitherto unsuspected, between the variational principle, the principle of reciprocity, and the differential scattering cross section. The use of Feynman diagrams in Appendix $C$ provides an intuitive understanding of some of these connections.

The integral equation for a homogeneous sphere with isotropic scatterers is shown to be separable, and the solution can be expanded in a multipole series. Detailed computations of the internal and photometric functions have been carried out. For all practical purposes, the optical properties of the homogeneous sphere of arbitrary size with isotropic scatterers are now known (or easily calculable). Our technique can be used to study the photometry of planetary atmospheres, cometary atmospheres, and the twilight problem in the Earth's atmosphere. These studies will be pursued and reported in future publications.

We thank K. S. K. Chow, L. W. Esposito, A. J. Friedson, R. M. Goody, and N. D. Sze for helpful discussions. The search for a variational principle for the planetary problem was assigned as one of the research topics in Professor R. M. Goody's class on atmospheric radiation at Harvard in 1972. The length of time it has taken to solve the problem is perhaps a measure of the sagacity of the master and the stupidity of the pupil (Y. L. Y.). The reasearch is supported by NASA grant NSG 7376 under the Planetary Atmospheres Program.

\section{APPENDIX A}

List of important photometric functions for the scattering of a parallel light beam by a particle or a planet. These functions are evaluated in terms of functions defined in this work (see Fig. $10 b$ for explanation of scattering angle $\alpha_{s}$ and phase angle $\alpha_{p}$ ). Note that $Q_{\text {sca }}=A$.

\section{PARTICLE PHOTOMETRIC FUNCTIONS}

Differential cross section:

$$
\frac{d \sigma}{d \Omega}\left(\alpha_{s}\right)=\frac{\omega_{0}}{\alpha^{2}} \sum_{l=0}^{\infty}(-1)^{l} f_{l} P_{l}\left(\cos \alpha_{s}\right)
$$

Scattering cross section:

$$
\sigma_{\mathrm{sca}}=2 \pi \int_{0}^{\pi} \frac{d \sigma}{d \Omega} \sin \alpha_{s} d \alpha_{s}=\frac{4 \pi \omega_{0}}{\alpha^{2}} f_{0}
$$

Scattering efficiency:

$$
Q_{\text {sca }}=\frac{\sigma_{\text {sca }}}{\pi a^{2}}=\frac{4 \omega_{0}}{\tau_{0}^{2}} f_{0}
$$

Extinction efficiency:

$$
Q_{\mathrm{ext}}=\frac{16}{\tau_{0}^{2} F_{0}} \int_{0}^{\tau_{0}} d \tau \tau S_{0}(\tau)
$$

Asymmetry factor:

$$
g=\frac{2 \pi}{\sigma_{\text {sca }}} \int_{0}^{\pi} \frac{d \sigma}{d \Omega} \sin \alpha_{s} \cos \alpha_{s} d \alpha_{s}=-\frac{f_{1}}{3 f_{0}}
$$

Phase function:

$$
p\left(\cos \alpha_{s}\right)=\frac{4 \pi \omega_{0}}{\sigma_{\text {sca }}} \frac{d \sigma}{d \Omega}\left(\alpha_{s}\right)=\frac{\omega_{0}}{f_{0}} \sum_{l=0}^{\infty}(-1)^{l} f_{l} P_{l}\left(\cos \alpha_{s}\right)
$$

\section{PLANETARY PHOTOMETRIC FUNCTIONS}

Flux :

$$
j\left(\alpha_{p}\right)=\frac{\pi F_{0}}{a^{2}} \frac{d \sigma}{d \Omega}\left(\alpha_{s}\right)=\frac{\omega_{0} \pi F_{0}}{\tau_{0}^{2}} \sum_{l=0}^{\infty} f_{l} P_{l}\left(\cos \alpha_{p}\right) .
$$


Geometric albedo:

$$
p=\frac{j(o)}{\pi F_{0}}=\frac{\omega_{0}}{\tau_{0}^{2}} \sum_{l=0}^{\infty} f_{l} .
$$

Phase variation:

$$
\varphi\left(\alpha_{p}\right)=\frac{j\left(\alpha_{p}\right)}{j(o)}=\frac{\sum_{l=0}^{\infty} f_{l} P_{l}\left(\cos \alpha_{p}\right)}{\sum_{l=0}^{\infty} f_{l}}
$$

Phase integral :

$$
q=2 \int_{0}^{\pi} d \alpha_{p} \varphi\left(\alpha_{p}\right)=\frac{4 f_{0}}{\sum_{l=0}^{\infty} f_{l}} .
$$

Bond albedo:

$$
A=p q=\frac{4 \omega_{0} f_{0}}{\tau_{0}^{2}}
$$

\section{APPENDIX B}

\section{DIFFERENTIAL CROSS SECTION}

The definition of the differential cross section given by equation (5) is motivated by similar definitions in the electromagnetic and quantum theories of scattering (Jackson 1975, p. 412; Landau and Lifshitz 1965, pp. 469-472). Since this is the first time that this quantity is introduced in radiative transfer, we shall give examples demonstrating the connection between the differential cross section and related physical quantities which may be more familiar to the reader.

In an optically thin homogeneous medium equation (5) can be evaluated in the single scattering approximation

$$
\frac{d \sigma}{d \Omega}\left(\boldsymbol{k}, \boldsymbol{k}_{0}\right) \approx \frac{1}{4 \pi} \alpha V P\left(\boldsymbol{k}, \boldsymbol{k}_{0}\right)
$$

The total cross section in this case is

$$
\sigma \approx \alpha \omega_{0} V=N_{0} \omega_{0} \sigma_{0}
$$

where $N_{0}$ is the total number of scatterers each with cross section $\sigma_{0}$.

For scattering of sunlight by a plane-parallel atmosphere of area $A, A$ being large, equation (5) gives

$$
\lim _{A \rightarrow \infty} \frac{\pi}{A} \frac{d \sigma}{d \Omega}\left(\mu, \mu_{0}\right)=\mu \mu_{0} R\left(\mu, \mu_{0}\right),
$$

where the reflectivity $R\left(\mu, \mu_{0}\right)$ is as defined by van de Hulst (1980a, pp. 46-50), and the meaning of the relevant angles are as shown in Figure $10 a$.

For scattering of sunlight by a sphere of radius $a$ (see Fig. $10 b$ for description of geometry), evaluation of equation (5) yields

$$
\frac{d \sigma}{d \Omega}\left(\alpha_{s}\right)=\frac{a^{2}}{\pi F_{0}} j\left(\alpha_{p}\right)
$$

where $\alpha_{s}$ is the scattering angle given by $\cos \alpha_{s}=\boldsymbol{k} \cdot \boldsymbol{k}_{0}, \alpha_{p}=\pi-\alpha_{s}$ and $j\left(\alpha_{p}\right)$ is a well-known photometric function, the flux (Horak 1950; Harris 1961).

It is of special interest to evaluate equation (B4) for the simple case of a Lambert sphere with surface reflectivity $\lambda$. From van de Hulst $(1980 a, b)$ we have

$$
j\left(\alpha_{p}\right)=\frac{2}{3} \lambda F_{0}\left[\sin \alpha_{p}+\left(\pi-\alpha_{p}\right) \cos \alpha_{p}\right]=\frac{2}{3} \lambda F_{0}\left(\sin \alpha_{s}-\alpha_{s} \cos \alpha_{s}\right) .
$$

Substituting equation (B5) into equation (B4), we obtain

$$
\frac{d \sigma}{d \Omega}\left(\alpha_{s}\right)=\frac{2 \lambda}{3 \pi} \alpha^{2}\left(\sin \alpha_{s}-\alpha_{s} \cos \alpha_{s}\right) .
$$

On integrating equation (B6) we get the expected total cross section

$$
\sigma_{\mathrm{sca}}=\lambda \pi a^{2} \text {. }
$$


a)

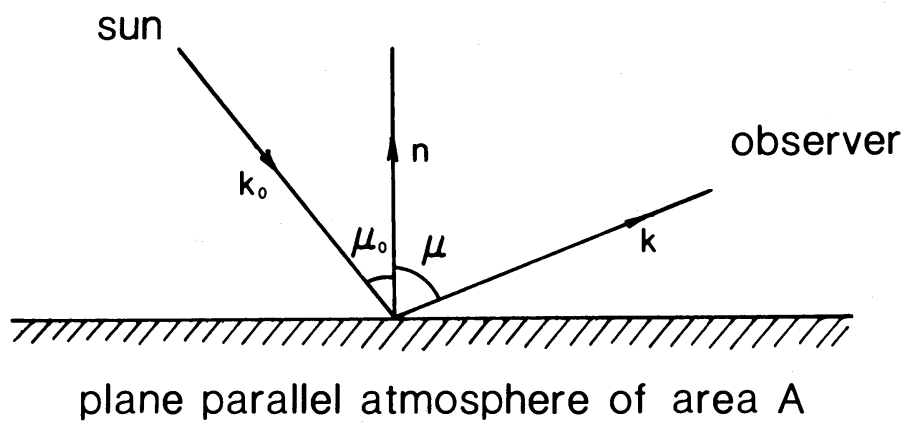

b)

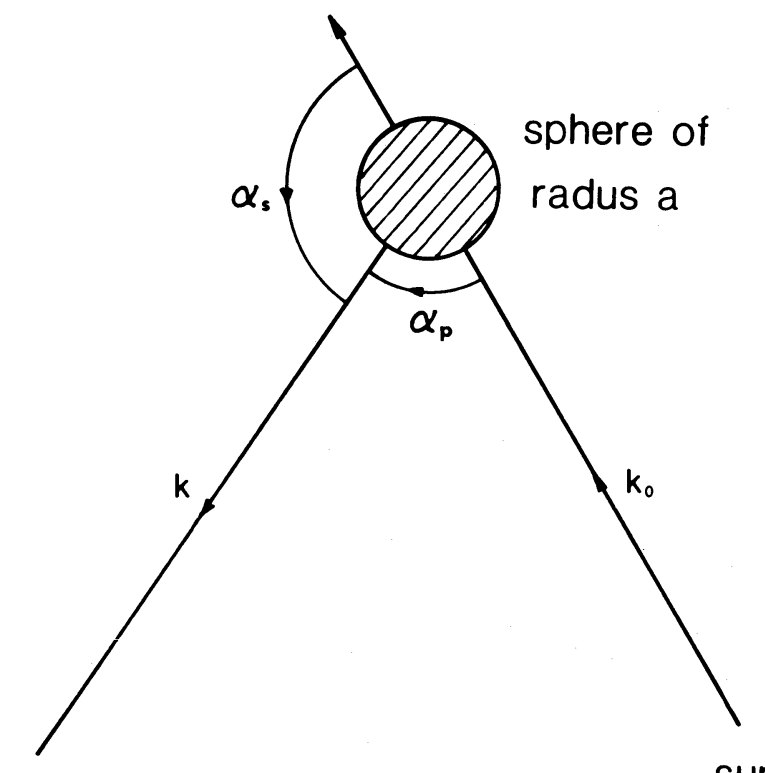

\section{observer}

sun

FIG. 10.-(a) Schematic diagram for scattering of sunlight by a plane-parallel atmosphere of area $A$ and locan normal $\boldsymbol{n}$. The cosine of the zenith angles $\mu_{0}$ and $\mu$ are given by $-\boldsymbol{k}_{0} \cdot \boldsymbol{n}$ and $\boldsymbol{k} \cdot \boldsymbol{n}$, respectively. (b) Schematic diagram for scattering of sunlight by a sphere of radius $a$. The phase angle $\alpha_{p}$ and the scattering angle $\alpha_{s}$ are defined by $\cos \alpha_{p}=-\boldsymbol{k}_{0} \cdot \boldsymbol{k}$ and $\cos \alpha_{s}=\boldsymbol{k}_{0} \cdot \boldsymbol{k}$.

\section{APPENDIX C}

\section{METHOD OF SUCCESSIVE ORDERS}

To gain insight into the connection between the microscopic symmetry of $P\left(\boldsymbol{r} ; \mathbf{\Omega}_{\mathbf{1}}, \mathbf{\Omega}_{2}\right)$ and the macroscopic symmetry of $(d \sigma / d \mathbf{\Omega})$ $\left(\boldsymbol{k}_{1}, \boldsymbol{k}_{2}\right)$, we consider the method of successive orders for solving equation (1) (van de Hulst 1980a, pp. 46-50). The idea is simple. We can regard equation (1) as an iterative equation for keeping track of photons that have been scattered once, twice, ..., $i$ times, .... By summing over the contribution from all orders we obtain the correct solution to equation (1). The advantage of this approach is the intuitive understanding it offers. The main disadvantage seems to be slow convergence (Irvine 1975), but that is not the issue here, since we are primarily interested in gaining theoretical insight.

Since radiative transfer is a simple many-body problem, it is instructive to represent the physical processes using Feynman diagrams. Equations (1) and (5) contain four fundamental processes: the input factor $\epsilon(\boldsymbol{r}, \boldsymbol{k})$, the output factor $\epsilon(\boldsymbol{r},-\boldsymbol{k})$, the distributor $[\alpha(\boldsymbol{r}) / 4 \pi] P\left(\boldsymbol{r} ; \boldsymbol{\Omega}_{1}, \boldsymbol{\Omega}_{2}\right)$, and the propagator $\pi\left(\boldsymbol{r}_{1}, \boldsymbol{r}_{2}\right)$, as summarized in Table 2 using symbols adapted from the quantum theory of many-body problems (Mahan 1981). Applying the method of successive orders we can write the differential cross section as a series of partial cross sections:

$$
\frac{d \sigma}{d \Omega}\left(\boldsymbol{k}_{2}, \boldsymbol{k}_{1}\right)=\sum_{i=1}^{\infty} \frac{d \sigma^{(i)}}{d \Omega}\left(\boldsymbol{k}_{2}, \boldsymbol{k}_{1}\right),
$$

where the superscript $i$ refers to the number of times the photon has been scattered. The expressions for the partial cross sections can be easily obtained with the aid of Feynman diagrams. For single scattering we have

$$
\frac{d \sigma^{(1)}}{d \Omega}\left(\boldsymbol{k}_{2}, \boldsymbol{k}_{1}\right)=\left\langle\epsilon\left(\boldsymbol{r},-\boldsymbol{k}_{2}\right) \Lambda\left(\boldsymbol{r}, \boldsymbol{k}_{2}, \boldsymbol{k}_{1}\right) \epsilon\left(\boldsymbol{r}, \boldsymbol{k}_{1}\right)\right\rangle
$$


TABLE 2

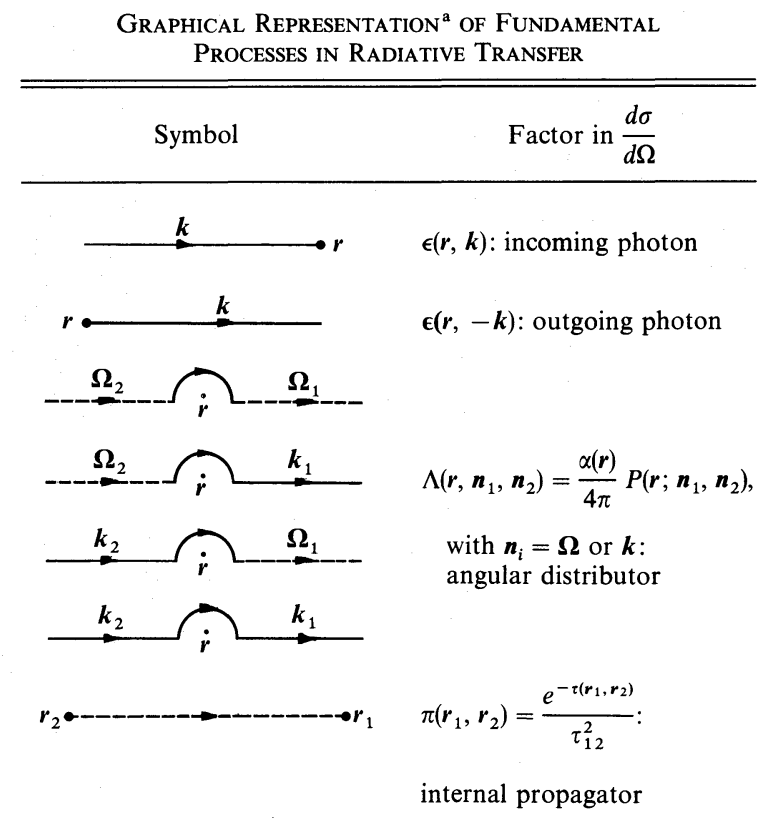

a These symbols are used in the construction of Feynman diagrams in Fig. 11.

where the angle brackets imply integration over internal variables. The reverse process is

$$
\frac{d \sigma^{(1)}}{d \Omega}\left(-\boldsymbol{k}_{1},-\boldsymbol{k}_{2}\right)=\left\langle\epsilon\left(\boldsymbol{r}, \boldsymbol{k}_{1}\right) \Lambda\left(\boldsymbol{r},-\boldsymbol{k}_{1},-\boldsymbol{k}_{2}\right) \epsilon\left(\boldsymbol{r},-\boldsymbol{k}_{2}\right)\right\rangle .
$$

The expressions (C2) and (C3) are represented by the Feynman diagrams in Figure 11a. For photons that are scattered twice, we have

$$
\frac{d \sigma^{(2)}}{d \Omega}\left(\boldsymbol{k}_{2}, \boldsymbol{k}_{1}\right)=\left\langle\epsilon\left(\boldsymbol{r}_{2},-\boldsymbol{k}_{2}\right) \Lambda\left(\boldsymbol{r}_{2}, \boldsymbol{k}_{2}, \hat{\boldsymbol{r}}_{21}\right) \pi\left(\boldsymbol{r}_{2}, \boldsymbol{r}_{1}\right) \Lambda\left(\boldsymbol{r}_{1}, \hat{\boldsymbol{r}}_{21}, \boldsymbol{k}_{1}\right) \epsilon\left(\boldsymbol{r}_{1}, \boldsymbol{k}_{1}\right)\right\rangle,
$$

where the angle brackets have the same meaning as before. The reverse process is

$$
\frac{d \sigma^{(2)}}{d \Omega}\left(-\boldsymbol{k}_{1},-\boldsymbol{k}_{2}\right)=\left\langle\epsilon\left(\boldsymbol{r}_{1}, \boldsymbol{k}_{1}\right) \Lambda\left(\boldsymbol{r}_{1},-\boldsymbol{k}_{1}, \hat{\boldsymbol{r}}_{12}\right) \pi\left(\boldsymbol{r}_{1}, \boldsymbol{r}_{2}\right) \Lambda\left(\boldsymbol{r}_{2}, \hat{\boldsymbol{r}}_{12},-\boldsymbol{k}_{2}\right) \epsilon\left(\boldsymbol{r}_{2},-\boldsymbol{k}_{2}\right)\right\rangle,
$$

where $\hat{\boldsymbol{r}}_{12}=-\hat{\boldsymbol{r}}_{21}$. The corresponding Feynman diagrams are shown in Figure $11 b$. Inspection of equations (C2)-(C5) reveals that

$$
\frac{d \sigma^{(i)}}{d \Omega}\left(-\boldsymbol{k}_{1},-\boldsymbol{k}_{2}\right)=\frac{d \sigma^{(i)}}{d \Omega}\left(\boldsymbol{k}_{2}, \boldsymbol{k}_{1}\right)
$$

for $i=1,2$ if

$$
\Lambda\left(\boldsymbol{r},-\mathbf{\Omega}_{2},-\mathbf{\Omega}_{1}\right)=\Lambda\left(\boldsymbol{r}, \mathbf{\Omega}_{1}, \mathbf{\Omega}_{2}\right)
$$

and

$$
\pi\left(\boldsymbol{r}_{2}, \boldsymbol{r}_{1}\right)=\pi\left(\boldsymbol{r}_{1}, \boldsymbol{r}_{2}\right) .
$$

Equation $(\mathrm{C} 8)$ is true from its definition. Equation $(\mathrm{C} 7)$ holds if

$$
P\left(\boldsymbol{r} ;-\mathbf{\Omega}_{2},-\mathbf{\Omega}_{1}\right)=P\left(\boldsymbol{r} ; \mathbf{\Omega}_{1}, \mathbf{\Omega}_{2}\right) .
$$

We can now see the direct connection between equations (C9) and (C6). The argument can obviously be generalized to all orders, and, hence, equation (C9) implies the reciprocity symmetry of the differential cross section (eq. [C1]). The results obtained here are stronger than those in $\S$ III for two reasons: the symmetry requirement (eq. [C9]) is weaker than equation (6), and the reciprocity relation (eq. [C6]) is true for each order of scattering as well as the sum total given by equation (39).

The method of successive orders offers a demonstration of the advantage of the functional expression (eq. [39]) over the direct expression (eq. [27]) for approximate evaluation of the differential cross section. Let $J_{n}$ and $\tilde{J}_{n}$ be approximate solutions of 


\section{SHIA AND YUNG}
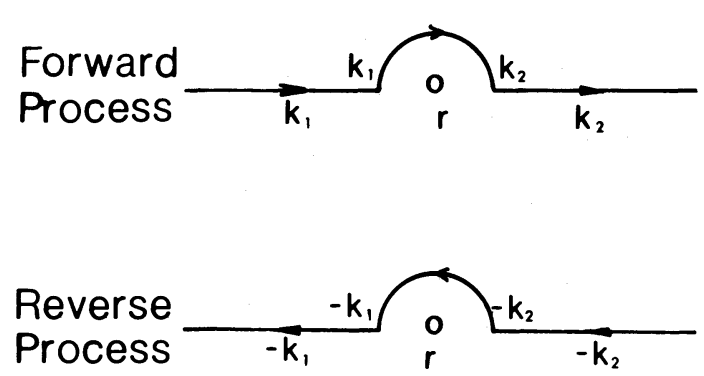

\section{a) photon scattered once}
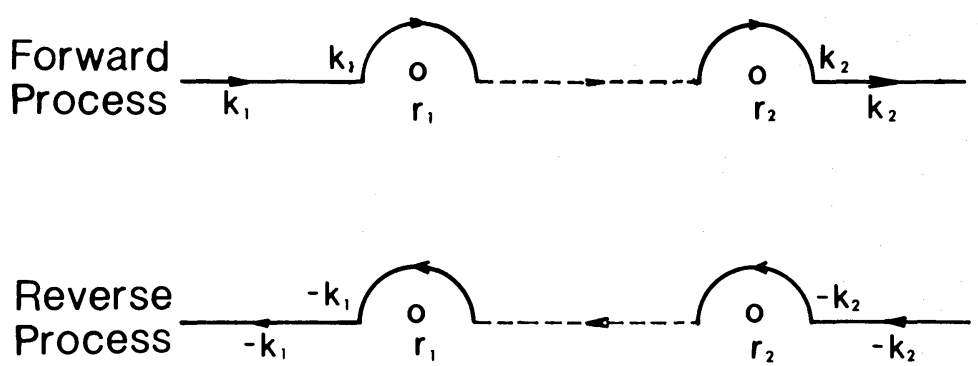

\section{b) photon scattered twice}

FIG. 11. - (a) Feynman diagrams for photons that are scattered once. See Table 2 for explanation of symbols. (b) Same as $(a)$ for photons that are scattered twice.

equations (25) and (26) that are correct to $n$th order; i.e.,

$$
\begin{aligned}
& J_{n}=\sum_{i=1}^{n} J^{(n)}, \\
& \tilde{J}_{n}=\sum_{i=1}^{n} \widetilde{J}^{(n)},
\end{aligned}
$$

where $J^{(i)}$ and $\tilde{J}^{(i)}$ refer to the radiation field of photons which are scattered $i$ times. It can be shown, after some tedious but straightforward algebra, that expressions (27) and (39), respectively, give

$$
\begin{aligned}
& \frac{d \sigma}{d \Omega}\left(\boldsymbol{k}_{2}, \boldsymbol{k}_{1}\right)=\sum_{i=1}^{n} \frac{d \sigma^{(i)}}{d \Omega}\left(\boldsymbol{k}_{2}, \boldsymbol{k}_{1}\right), \\
& \frac{d \sigma}{d \Omega}\left(\boldsymbol{k}_{2}, \boldsymbol{k}_{1}\right)=\sum_{i=1}^{2 n} \frac{d \sigma^{(i)}}{d \Omega}\left(\boldsymbol{k}_{2}, \boldsymbol{k}_{1}\right) .
\end{aligned}
$$

Thus the functional expression achieves an accuracy equivalent to $2 n$ orders of scattering for approximate radiation fields that are correct only to $n$ orders of scattering.

\section{APPENDIX D}

\section{GREEN'S FUNCTION}

To derive equation (51), all we need is to use the following formulae (Abramowitz and Stegun 1972, pp. 437-439):

$$
\begin{aligned}
j_{l}(z) & =h_{l}^{(1)}(z)+h_{l}^{(2)}(z) \\
h_{l}^{(1)}(z) & =i^{-l-1} z^{-1} e^{i z} \sum_{k=0}^{l} \frac{(l+k) !}{k !(l-k) !}(-2 i z)^{-k} \\
h_{l}^{(2)}(z) & =i^{l+1} z^{-1} e^{-i z} \sum_{k=0}^{l} \frac{(l+k) !}{k !(l-k) !}(2 i z)^{-k}
\end{aligned}
$$


TABLE 3

VAlues of Coefficients $^{\mathrm{a}} A_{l m}$ AND $B_{l m}$ FOR the First Three Multipoles $l=0,1$, AND 2

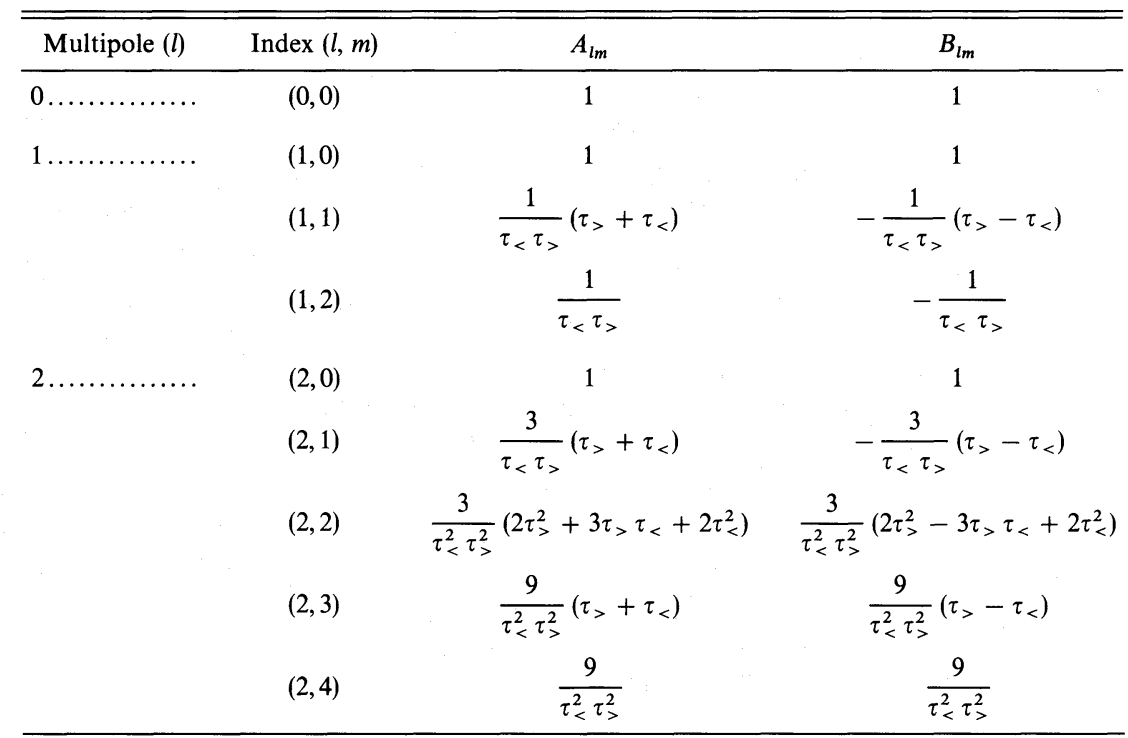

${ }^{a}$ Defined in eq. (51).

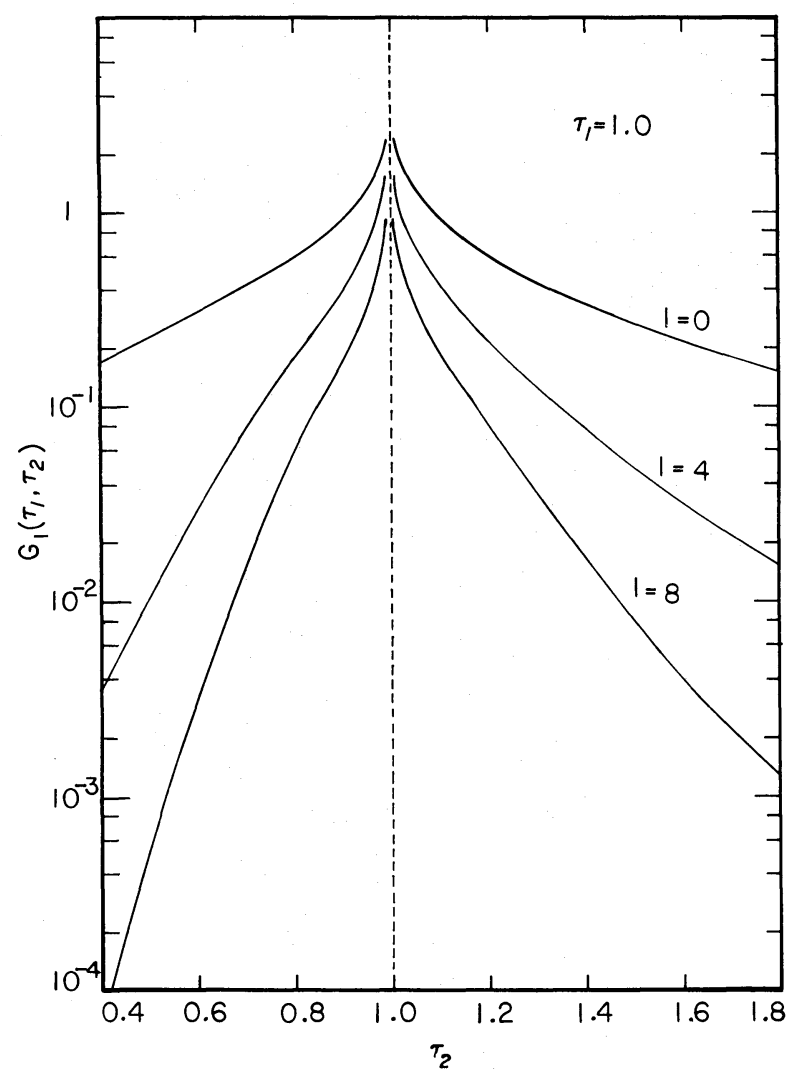

FIG. $12 a$

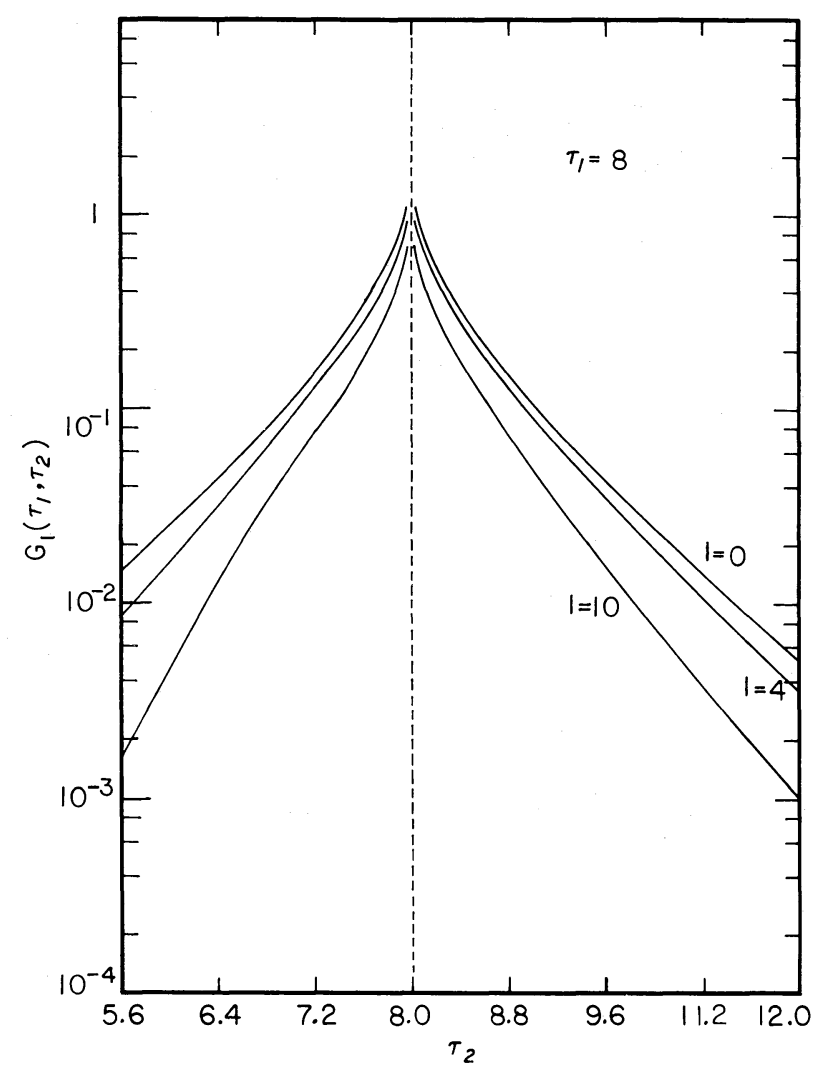

FIG. $12 b$

Fig. 12.-Green's function $G_{l}\left(\tau_{1}, \tau_{2}\right)$ as a function of $\tau_{2} .(a) l=0,4,8$ and $\tau_{1}=1.0 .(b) l=0,4,8$ and $\tau_{1}=8.0$. 


\section{SHIA AND YUNG}

The resulting expression in equation (51) follows from simplifying and collecting the coefficients for the exponential integral functions. Representative values for the coefficients $A_{l m}$ and $B_{l m}$ are shown in Table 3. Equation (51) is unfortunately not suitable for numerical computation of $G_{l}\left(\tau_{1}, \tau_{2}\right)$ when $l$ is large and $\tau_{1}$ and $\tau_{2}$ are $\lesssim 1$. The reason is that in this situation the individual terms in equation (51) are extremely large even though they eventually cancel each other to yield a much smaller sum. Indeed, round-off errors render equation (51) useless for $l \geq 10$ even for computations carried out with 32 significant figures. In practice we use equation (51) only for $l=0-2$. For other multipoles we use another formula:

$$
G_{l}\left(\tau_{1}, \tau_{2}\right)=\frac{\tau_{1} \tau_{2}}{2} \int_{-1}^{1} \frac{e^{-\left(\tau_{1}^{2}+\tau_{2}^{2}-2 \tau_{1} \tau_{2} \mu\right)^{1 / 2}}}{\tau_{1}^{2}+\tau_{2}^{2}-2 \tau_{1} \tau_{2} \mu} P_{l}(\mu) d \mu
$$

and carry out the numerical evaluation with a 36 point Gaussian quadrature. The effect of the singularity $G_{l}(\tau, \tau)$ is properly assessed by its integral over a grid box (Yung 1976).

Figure 12 shows the values of $G_{l}\left(\tau_{1}, \tau_{2}\right)$ for two representative cases: $\tau_{1}=1$ and $\tau_{1}=8$. It is of interest to note that as $l$ increases Green's functions become more concentrated near $\tau_{1}=\tau_{2}$, and the areas under the curves become smaller. The net effect of this behavior is to make the first term more important relative to the second term on the right-hand side of equation (50). Hence, it would be practical to use the method of successive orders for solving equation (50) for higher multipoles.

\section{REFERENCES}

Abramowitz, M., and Stegun, I. A. 1972, Handbook of Mathematical Functions (Washington, DC: NBS).

Adams, C. N., and Kattawar, G. W. 1978, Icarus, 35, 139.

Anderson, D. E., Jr. 1983, Planet. Space Sci., 31, 1517.

Anderson, D. E., Jr., and Hord, C. W. 1977, Planet. Space Sci., 25, 563

Bellman, R. E., Kagiwada, H. H., Kalaba, R. E., and Ueno, S. 1969, Icarus, 11, 417.

Case, K. M. 1957, Rev. Mod. Phys., 29, 651.

Case, K. M., and Zweifel, P. F. 1967, Linear Transport Theory (Reading, MA: Addison-Wesley).

Cercignani, C. 1969, in Transport Theory, ed. R. Bellman, G. Birkhoff, and Abu-Shumays (Providence, RI: American Mathematical Society), p. 249.

Cercignani, C., and Pagani, C. D. 1966, Phys. Fluids, 9, 1167.

Chandrasekhar, S. 1960, Radiative Transfer (New York: Dover).

Chang, K. M., and Kylafis, N. D. 1983, Ap. J., 265, 1005.

Cheyney, H., III, and Arking, A. 1976, Ap. J., $207,808$.

Chow, K. S. K., Friedson, A. J., and Yung, Y. L. 1984, Iracus, 59, 177.

Cochran, W. D. 1977, Icarus, 31, 325.

Davison, B. 1957, Neutron Transport Theory (Oxford: Oxford University Press).

Debye, P. 1909, Ann. Phys., 30, 57.

Flannery, B. P., Roberge, W., and Rybicki, G. B. 1980, Ap. J., 236, 598

Fouquart, Y., Irvine, W. M., and Lenoble, J., eds. 1980, Standard Procedures to Compute Atmospheric Radiative Transfer in a Scattering Atmosphere (Boulder: National Center for Atmospheric Research).

Francis, N. C., Stewart, J. C., Bohl, L. S., and Kreiger, T. J. 1959, Progr. Nucl. Eng., Ser. 1, 3, 517

Gerjuoy, E., Rau, A. R. P., and Spruch, L. 1983, Rev. Mod. Phys., 55, 725.

Gerjuoy, E., and Saxon, D. S. 1954, Phys. Rev., 94, 1445.

Hanner, M. S. 1979, in IAU Symposium 90 , Solid Particles in the Solar System, ed. I. Halliday and B. A. McIntosh (Dordrecht: Reidel), p. 223.

Hansen, J. E. 1969, J. Atmos. Sci., 26, 478.

Harris, D. L. 1961, in The Solar System, Vol. 3, Planets and Satellites, ed. G. P. Kuiper and B. M. Middlehurst (Chicago: University of Chicago Press), p. 272 .

Note added in proof.-The authors thank Professor van de Hulst for kindly pointing out that the $g$ for an infinite isotropically scattering spherical atmosphere is

$$
\frac{1}{2}-\frac{4}{3} q_{\infty}=-0.4472614530
$$

Run-LIE SHIA: Division of Physics, Mathematics, and Astronomy, California Institute of Technology, Pasadena, CA 91125

YUK L. YUNG: Division of Geological and Planetary Sciences, 170-25, California Institute of Technology, Pasadena, CA 91125
Hellmich, R. 1981, Astr. Ap., 93, 341.

Horak, H. G. 1950, Ap. J., 112, 445.

Hovenier, J. W. 1969, J. Atmos. Sci., 26, 488.

.

Jackson, J. D. 1975, Classical Electrodynamics (New York: Wiley).

Kattawar, G. W. 1975, J. Quant. Spectrosc. Rad. Transf., 15, 839.

Landau, L. D., and Lifshitz, E. M. 1965, Quantum Mechanics (New York: Pergamon).

Levin, H., and Schwinger, J. 1950, Comm. Pure Appl. Math., 3, 355.

Lippmann, B. A., and Schwinger, J. 1950, Phys. Rev., 79, 469.

Mahan, G. D. 1981, Many-Particle Physics (New York: Plenum)

Mie, G. 1908, Ann. Phys. 25, 377.

Minnaert, M. 1941, Ap. J., 93, 403.

Ney, E. P., and Merrill, K. M. 1976, Science, 194, 1051.

Peierls, R. 1939, Proc, Cambridge Phil. Soc, 35, 610.

Pomraning, G. C., and Clark, M., Jr. 1963a, Nucl. Sci. Eng., 16, 147.

Sato 1963 , Nucl. Sci. Eng., 16, 155.

Smith., and Hansen, J. E. 1979, J. Atmos. Sci., 36, 1133.

Sobolev, V. V. 1972 , Astrofizika, 8, 117.

. 1975, Light Scattering in Planetary Atmospheres (Oxford: Pergamon),

Stokes, R. A., and DeMarcus, W. C. 1971, Icarus, 14, 307.

Sze, N. D. 1976, J. Quant. Spectrosc. Rad. Transf., 16, 763.

Tomasko, M. G., and Doose, L. R. 1984, Icarus, 58, 1.

Tomasko, M. G., West, R. A., and Castillo, N. D. 1978, Icarus, 33, 558.

van de Hulst, 1957, Light Scattering by Small Particles (New York: Wiley) 1980 a, Multiple Light Scattering, Vol. 1 (New York: Academic). $1980 b$ Multiple Light Scattering, Vol 2 (New York: Academic)

Yung, Y. L. 1976, J. Quant. Spectrosc. Rad. Transf., 16, 755.
Modali, S. B., Brandt, J. C., and Kastner, S. O. 1975, Ap. J., 199, 530. 